The Astrophysical Journal, 675:626-643, 2008 March 1

(C) 2008. The American Astronomical Society. All rights reserved. Printed in U.S.A.

\title{
OPTICAL AND NEAR-INFRARED OBSERVATIONS OF THE HIGHLY REDDENED, RAPIDLY EXPANDING TYPE Ia SUPERNOVA SN 2006X IN M100
}

\author{
Xiaofeng Wang, ${ }^{1,2}$ Weidong Li, ${ }^{1}$ Alexei V. Filippenko, ${ }^{1}$ Kevin Krisciunas, ${ }^{3}$ Nicholas B. Suntzeff, ${ }^{3}$ \\ Junzheng Li, ${ }^{2}$ Tianmeng Zhang, ${ }^{4}$ Jingsong Deng, ${ }^{4}$ Ryan J. Foley, ${ }^{1}$ Mohan Ganeshalingam, ${ }^{1}$ \\ Tipei Li, ${ }^{2}$ YuQing Lou, ${ }^{2}$ Yulei Qiu, ${ }^{4}$ Rencheng Shang, ${ }^{2}$ Jeffrey M. Silverman, ${ }^{1}$ \\ ShuANGnan Zhang, ${ }^{2}$ and Youhong Zhang ${ }^{2}$ \\ Received 2007 August 1; accepted 2007 November 11
}

\begin{abstract}
We present extensive optical (UBVRI), near-infrared $(J K)$ light curves and optical spectroscopy of the Type Ia supernova SN 2006X in the nearby galaxy NGC 4321 (M100). Our observations suggest that either SN 2006X has an intrinsically peculiar color evolution or it is highly reddened $\left[E(B-V)_{\text {host }}=1.42 \pm 0.04 \mathrm{mag}\right]$ with $R_{V}=$ $1.48 \pm 0.06$, much lower than the canonical value of 3.1 for the average Galactic dust. SN 2006X also has one of the highest expansion velocities ever published for an SN Ia. Compared with the other SNe Ia we analyzed, SN 2006X has a broader light curve in the $U$ band, a more prominent bump/shoulder feature in the $V$ and $R$ bands, a more pronounced secondary maximum in the $I$ and NIR bands, and a remarkably smaller late-time decline rate in the $B$ band. The $B-V$ color evolution shows an obvious deviation from the Lira-Phillips relation at 1-3 months after maximum brightness. At early times, optical spectra of SN 2006X displayed strong, high-velocity features of both intermediatemass elements ( $\mathrm{Si}, \mathrm{Ca}$, and $\mathrm{S}$ ) and iron peak elements, while at late times they showed a relatively blue continuum, consistent with the blue $U-B$ and $B-V$ colors at similar epochs. A light echo and/or the interaction of the SN ejecta and its circumstellar material may provide a plausible explanation for its late-time photometric and spectroscopic behavior. Using the Cepheid distance of M100, we derive a Hubble constant of $72.8 \pm 8.2 \mathrm{~km} \mathrm{~s}^{-1} \mathrm{Mpc}^{-1}$ (statistical) from the normalized dereddened luminosity of SN 2006X. We briefly discuss whether abnormal dust is a universal signature for all $\mathrm{SNe}$ Ia and whether the most rapidly expanding objects form a subclass with distinct photometric and spectroscopic properties.
\end{abstract}

Subject headings: distance scale — dust, extinction — galaxies: individual (NGC 4321) supernovae: individual (SN 2006X)

\section{INTRODUCTION}

Type Ia supernovae (SNe Ia) are arguably the most accurate tools available for probing the expansion history of the universe, as their luminosities at maximum light can be calibrated to an uncertainty of $\sim 15 \%$ via several empirical relations between their luminosity and light/color curves (Wang et al. 2006d and references therein). Based on studies of SN Ia apparent brightness as a function of redshift, Riess et al. (1998) and Perlmutter et al. (1999) were the first to propose that the expansion of the universe is currently accelerating (for a review see, e.g., Filippenko 2005). This remarkable result, which suggests that the universe is primarily composed of mysterious dark energy, was subsequently confirmed by more studies using $\mathrm{SNe}$ Ia (Barris et al. 2004; Tonry et al. 2003; Knop et al. 2003; Riess et al. 2004, 2007; Astier et al. 2006; Wood-Vasey et al. 2007) and by independent methods such as the power spectrum of fluctuations in the cosmic microwave background radiation (e.g., Spergel et al. 2003, 2007) and baryon acoustic oscillations (Eisenstein et al. 2005).

Despite the success of using SNe Ia as cosmological probes, a disconcerting fact is that details of the properties of SN Ia progenitors and their environments remain poorly understood.

\footnotetext{
${ }^{1}$ Department of Astronomy, University of California, Berkeley, CA 947203411; wangxf@astro.berkeley.edu.

2 Physics Department and Tsinghua Center for Astrophysics (THCA), Tsinghua University, Beijing 100084, China; wang_xf@mail.tsinghua.edu.cn.

3 Physics Department, Texas A\&M University, College Station, TX 77843.

${ }^{4}$ National Astronomical Observatories of China, Chinese Academy of Sciences, Beijing 100012, China.
}

It is generally believed that an SN Ia is produced by a carbonoxygen (C-O) white dwarf (WD) accreting matter from a companion star in a binary system. However, the nature of the companion star in the binary system is still unclear: it could be a main-sequence star, a subgiant, or an evolved red giant (Nomoto et al. 1997), or even another degenerate C-O WD (Iben \& Tutukov 1984; Webbink 1984).

Studying the absorption by dust toward SNe Ia may provide a unique way to peer into their local environments and hence set constraints on the properties of their progenitor system. If the dust enveloping the SNe is totally or partially produced by the progenitor evolution, the ratio of the total to selective absorption $\left[R_{V}=A_{V} / E(B-V)\right]$ may differ from that of normal interstellar dust (e.g., Wang 2005). Moreover, accurate determination of the absorption toward an SN Ia helps establish the accurate luminosity required for precision cosmology.

Several studies of global color fits to SNe Ia have suggested that the dust obscuring SNe Ia in their host galaxies may differ from the dust observed in the Milky Way (Riess et al. 1996; Phillips et al. 1999; Altavilla et al. 2004; Reindl et al. 2005; Wang et al. 2006d): the measured value of $R_{V}$ is often found to be smaller than the canonical Galactic value of 3.1. We measure an average $R_{V}=2.4 \pm 0.2$ from a compilation of published $R_{V}$ values. ${ }^{5}$ The sample of well-observed, highly extinguished SNe Ia is small but growing, offering the opportunity to study variations

\footnotetext{
${ }^{5}$ Methods that do not separate the intrinsic color variation and the host galaxy reddening from the observed color would give even lower $R$-values (Tripp 1997; Astier et al. 2006; Wang et al. 2006b).
} 


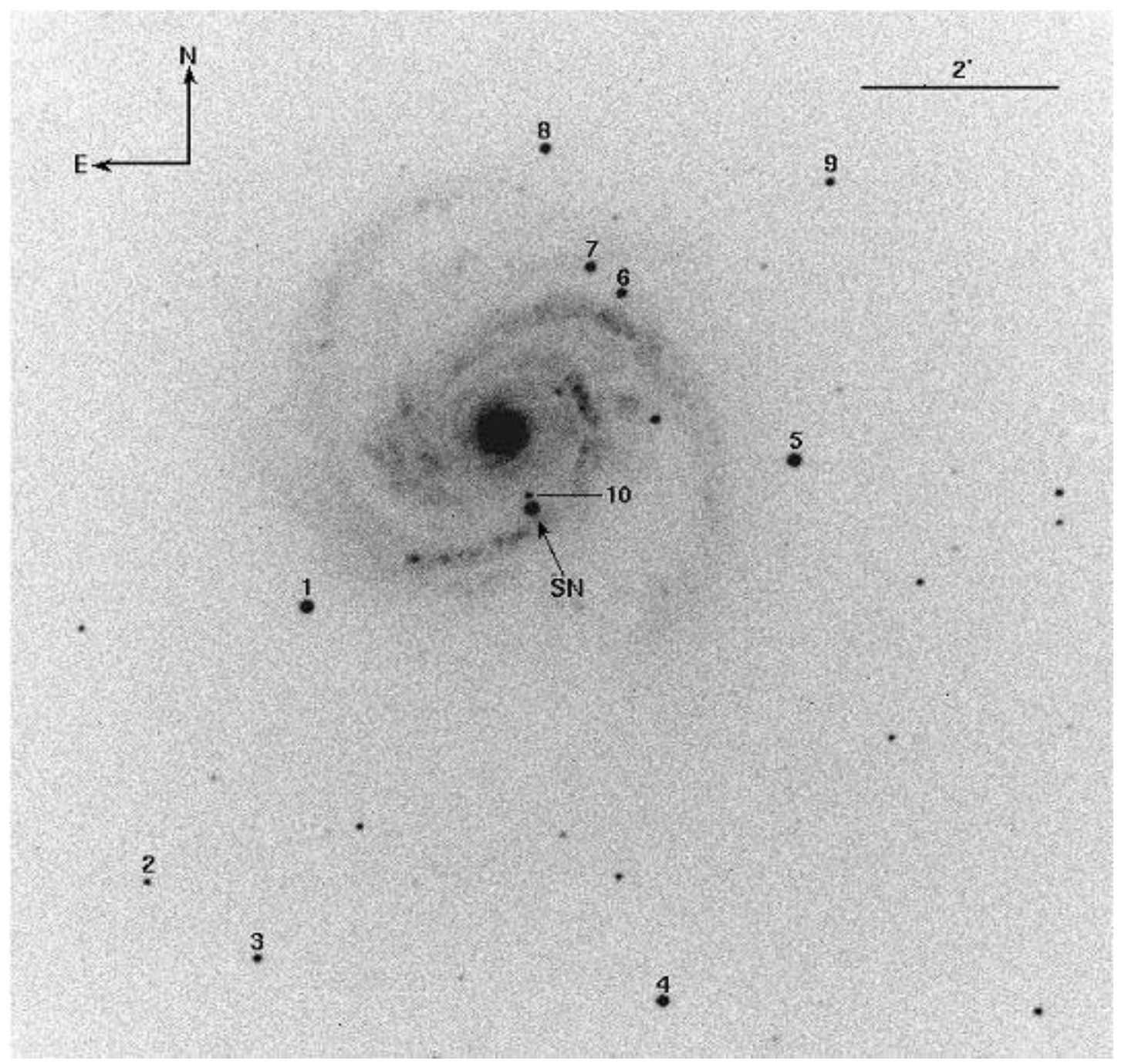

FIG. 1.-SN 2006X in NGC 4321 (M100). This is a $V$-band image taken by the $0.8 \mathrm{~m}$ TNT on 2006 February 19.69 . The supernova and 10 local reference stars (Table 2 ) are marked by numbers.

of the extinction law on an object-by-object basis. In almost all cases, $R_{V}$ is found to be smaller than 3.1 (Krisciunas et al. 2006; Elias-Rosa et al. 2006, hereafter ER06). It is interesting to investigate whether $R_{V}$ smaller than 3.1 is universal to all SNe Ia or just applies to a few or highly reddened events.

SN 2006X is a bright, nearby, highly reddened SN Ia and is favorable for a detailed study of reddening and dust properties. The recent report of variable, interstellar absorption $\mathrm{Na}$ I $\mathrm{D}$ lines in high-resolution spectra of SN 2006X indicates the presence of circumstellar material (CSM) around SN 2006X, which may be related to the stellar wind blown from the companion star (Patat et al. 2007). Here we present extensive photometric and spectroscopic observations of SN 2006X, enabling us to study its intervening dust and other characteristics. The paper is organized as follows. The observations and data reduction are described in $\S 2$, while $\S 3$ presents the UBVRIJK light curves, the color curves, and the extinction estimate. The spectral evolution is given in $\S 4$. We determine the distance and luminosity of the $\mathrm{SN}$ in $\S 5$. Our discussion and conclusions are given in $\S 6$.

\section{OBSERVATIONS AND DATA REDUCTION}

SN 2006X was discovered independently on 2006 February 7.10 (UT dates are used throughout this paper) by Suzuki \& Migliardi (2006), with J2000.0 coordinates $\alpha=12^{\mathrm{h}} 22^{\mathrm{m}} 53.90^{\mathrm{s}}$ and $\delta=15^{\circ} 48^{\prime} 32.90^{\prime \prime}$. It is $12^{\prime \prime}$ west and $48^{\prime \prime}$ south of the center of the nearby galaxy NGC 4321 (M100), a grand-design Sbc galaxy in the Virgo Cluster having a Cepheid distance of $30.91 \pm$ 0.14 mag (Freedman et al. 2001). A spectrum of SN 2006X taken shortly after its discovery shows it to be an early SN Ia (Quimby et al. 2006), similar to that of SN 2002bo 1-2 weeks before maximum light (Benetti et al. 2004) but with a redder continuum.

\subsection{Photometry}

Our photometric observations of SN 2006X began on 2006 February $8.15, \sim 11$ days before $B$-band maximum. Data were obtained with three telescopes: the $0.80 \mathrm{~m}$ THCA-NAOC Telescope (TNT) at Beijing Xinglong Observatory (BAO) in China, the $0.76 \mathrm{~m}$ Katzman Automatic Imaging Telescope (KAIT; Filippenko et al. 2001) at Lick Observatory in the US, and the $1.3 \mathrm{~m}$ telescope at Cerro Tololo Inter-American Observatory (CTIO) in Chile. The TNT observations were obtained using a $1340 \times 1300$ pixel back-illuminated charge-coupled device $(\mathrm{CCD})$ with a field of view (FOV) of $11.5^{\prime} \times 11.2^{\prime}\left(0.52^{\prime \prime}\right.$ pixel $\left.^{-1}\right)$, the KAIT observations were performed using an Apogee AP7 camera with an FOV of $6.6^{\prime} \times 6.6^{\prime}\left(0.79^{\prime \prime}\right.$ pixel $\left.^{-1}\right)$, and the CTIO $1.3 \mathrm{~m}$ observations were obtained using the dual-channel optical/near-infrared (NIR) camera ANDICAM having an 
TABLE 1

Color Terms for Different Telescopes

\begin{tabular}{|c|c|c|c|c|c|}
\hline Telescopes & $U$ & $B$ & $V$ & $R$ & $I$ \\
\hline 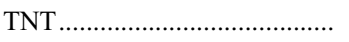 & $0.125 \pm 0.016$ & $-0.163 \pm 0.016$ & $0.078 \pm 0.007$ & $0.098 \pm 0.011$ & $-0.038 \pm 0.003$ \\
\hline 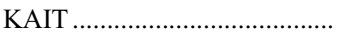 & $\ldots$ & $-0.053 \pm 0.013$ & $0.036 \pm 0.007$ & $0.069 \pm 0.009$ & $-0.001 \pm 0.007$ \\
\hline 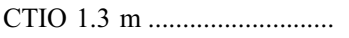 & $\ldots$ & $0.050 \pm 0.002$ & $-0.046 \pm 0.002$ & $-0.019 \pm 0.004$ & $-0.088 \pm 0.001$ \\
\hline
\end{tabular}

optical FOV of $6.3^{\prime} \times 6.3^{\prime}\left(0.37^{\prime \prime}\right.$ pixel $\left.^{-1}\right)$ and an IR FOV of $2.3^{\prime} \times 2.3^{\prime}\left(0.27^{\prime \prime} \mathrm{pixel}^{-1}\right)$. Broadband BVRI images were taken with all three telescopes, while the TNT also followed SN 2006X in the $U$ band and the CTIO $1.3 \mathrm{~m}$ telescope sampled the $J$ and $K$ bands.

As shown in Figure 1, SN 2006X is located between a relatively bright foreground star and the inner edge of one spiral arm of NGC 4321; thus, light contamination needs to be taken into account when measuring the SN flux. One way to do this is to apply galaxy subtraction. Template images of NGC 4321 in various bands were taken with KAIT and TNT on 2007 March 14 and 16 , respectively, roughly $1 \mathrm{yr}$ after the discovery of SN 2006X. For the CTIO 1.3 m observations, we used preexplosion $B$ and $V$ images of NGC 4321 obtained on 2000 April 24 with the Apache Point Observatory $3.5 \mathrm{~m}$ telescope in New Mexico.

To perform image subtraction, the image containing SN 2006X is first geometrically registered to the corresponding template image. Next, the total fluxes of corresponding foreground stars in the $\mathrm{SN}$ and template images are compared, and an appropriate scale factor is applied to the SN image in order to match the template image. Also, the point-spread functions (PSFs) of the two images are convolved to match. The template is then subtracted from the SN image, leaving the $\mathrm{SN}$ with a background that is free from host galaxy contamination. The final step is to perform standard PSF-fitting (or aperture) photometry to measure the instrumental magnitudes for the SN and the local standard stars with the IRAF ${ }^{6}$ DAOPHOT package (Stetson 1987). From the late-time Hubble Space Telescope (HST) Advanced Camera for Survey (ACS) archival images (Proposal 10991 by A. Crotts 2006), we speculate that SN 2006X was $~ 22.0$ mag in $B$ and $\sim 21.5$ mag in $V$ about 1 yr after maximum. This lingering light barely affects the early-time photometry, but it probably re-

\footnotetext{
${ }^{6}$ IRAF, the Image Reduction and Analysis Facility, is distributed by the National Optical Astronomy Observatory, which is operated by the Association of Universities for Research in Astronomy (AURA), Inc., under cooperative agreement with the National Science Foundation (NSF).
}

sults in underestimating the luminosity of SN $2006 \mathrm{X}$ by $3 \%-6 \%$ when the SN becomes as faint as 18-19 mag.

The transformation from the instrumental magnitudes to the standard Johnson $U B V$ (Johnson et al. 1966) and Kron-Cousins $R I$ (Cousins 1981) systems is established by observing, during a photometric night, a series of Landolt (1992) standards covering a large range of air masses and colors. The color terms obtained on different photometric nights show some differences, and their average values are listed in Table 1 for the filters used in the TNT, KAIT, and CTIO $1.3 \mathrm{~m}$ observations of SN 2006X.

A total of 10 photometric nights ( 2 for TNT, 3 for KAIT, and 5 for the CTIO $1.3 \mathrm{~m}$ ) were used to calibrate 10 local standard stars in the field of SN 2006X. Table 2 lists the final calibrated $U B V R I$ magnitudes and their uncertainties, while Table 3 presents the NIR $J$ - and $K$-band magnitudes. Only one photometric night was used to do the $U$-band calibration, so the calibrated magnitudes may have larger errors. The NIR magnitudes of star 10 were measured on 14 nights with respect to the Persson et al. (1998) standards P9144 and LHS2397a. The measured $J$ and $K$ magnitudes are 0.07 mag brighter than the values from the Two Micron All Sky Survey (2MASS), which obtained $J=14.793$ (0.025) mag and $K=13.995$ (0.040) mag.

These local standard stars are then used to transform the instrumental magnitudes of SN 2006X to the standard UBVRIJK system, and the final results of the photometry are listed in Tables 3 and 4 . Note that the $B V J K$-band data shown for the CTIO $1.3 \mathrm{~m}$ telescope include the $S$-corrections (see details in $\S 3.1$ ), which are listed in Table 5. The estimated error shown in parentheses is a quadrature sum of uncertainties in the photometry and the calibrations. The main source of error comes from the photometry, caused by photon noise and uncertainties in the image subtraction.

\subsection{Spectroscopy}

Spectra of SN 2006X were primarily obtained with the $2.16 \mathrm{~m}$ telescope at BAO using the Cassegrain spectrograph and BAO Faint Object Spectrograph and Camera (BFOSC) and with the

TABLE 2

Magnitudes of the Photometric Standards in the Field of SN 2006X

\begin{tabular}{|c|c|c|c|c|c|c|c|c|c|}
\hline Star & $\begin{array}{c}\alpha \\
(\mathrm{J} 2000.0)\end{array}$ & $\begin{array}{c}\delta \\
(\mathrm{J} 2000.0)\end{array}$ & $U$ & $B$ & $V$ & $R$ & $I$ & $J$ & $K$ \\
\hline 1 1........................... & 122303.77 & 154731.6 & $14.91(02)$ & $14.758(007)$ & $14.123(003)$ & $13.759(005)$ & $13.393(006)$ & $\cdots$ & $\cdots$ \\
\hline $2 \ldots \ldots \ldots \ldots \ldots \ldots \ldots \ldots \ldots \ldots \ldots \ldots \ldots \ldots$ & 122310.68 & 154439.3 & $17.20(15)$ & $17.069(036)$ & $16.809(019)$ & $16.549(027)$ & $16.177(024)$ & $\ldots$ & $\ldots$ \\
\hline 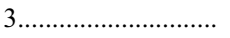 & 122305.93 & 154351.4 & $16.36(08)$ & $16.613(022)$ & $16.082(010)$ & $15.746(014)$ & $15.385(012)$ & $\ldots$ & $\ldots$ \\
\hline 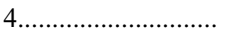 & 122248.29 & 154324.9 & $\ldots$ & $15.413(013)$ & $14.763(013)$ & $14.411(004)$ & $14.083(004)$ & $\ldots$ & $\ldots$ \\
\hline 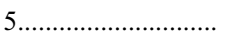 & 122242.60 & 154903.4 & $14.94(02)$ & $14.753(011)$ & $14.100(013)$ & $13.746(014)$ & $13.404(010)$ & $\ldots$ & $\ldots$ \\
\hline 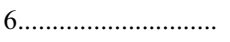 & 122250.09 & 155047.9 & $16.51(09)$ & $16.323(014)$ & $15.675(026)$ & $15.305(028)$ & $14.941(023)$ & $\ldots$ & $\ldots$ \\
\hline 7 & 122251.46 & 155104.5 & $16.75(09)$ & $16.340(020)$ & $15.458(021)$ & $14.945(008)$ & $14.445(008)$ & $\ldots$ & $\ldots$ \\
\hline 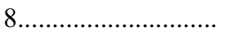 & 122253.40 & 155218.7 & $17.92(23)$ & $16.380(010)$ & $15.224(022)$ & $14.425(018)$ & $13.715(033)$ & $\ldots$ & $\ldots$ \\
\hline $9 \ldots \ldots \ldots \ldots \ldots \ldots \ldots \ldots \ldots$ & 122241.05 & 155157.8 & $16.67(09)$ & $16.665(008)$ & $16.131(010)$ & $15.775(013)$ & $15.430(005)$ & $\ldots$ & $\ldots$ \\
\hline $10 \ldots \ldots \ldots \ldots \ldots \ldots \ldots \ldots$ & 122253.98 & 154840.9 & $\ldots$ & $18.233(017)$ & $17.053(012)$ & $16.309(006)$ & $15.613(014)$ & $14.721(006)$ & $13.925(009)$ \\
\hline
\end{tabular}

Notes.-Units of right ascension are hours, minutes, and seconds, and units of declination are degrees, arcminutes, and arcseconds. See Fig. 1 for a chart of SN $2006 \mathrm{X}$ (in M100) and the comparison stars. 
TABLE 3

Optical Photometry of SN 2006X

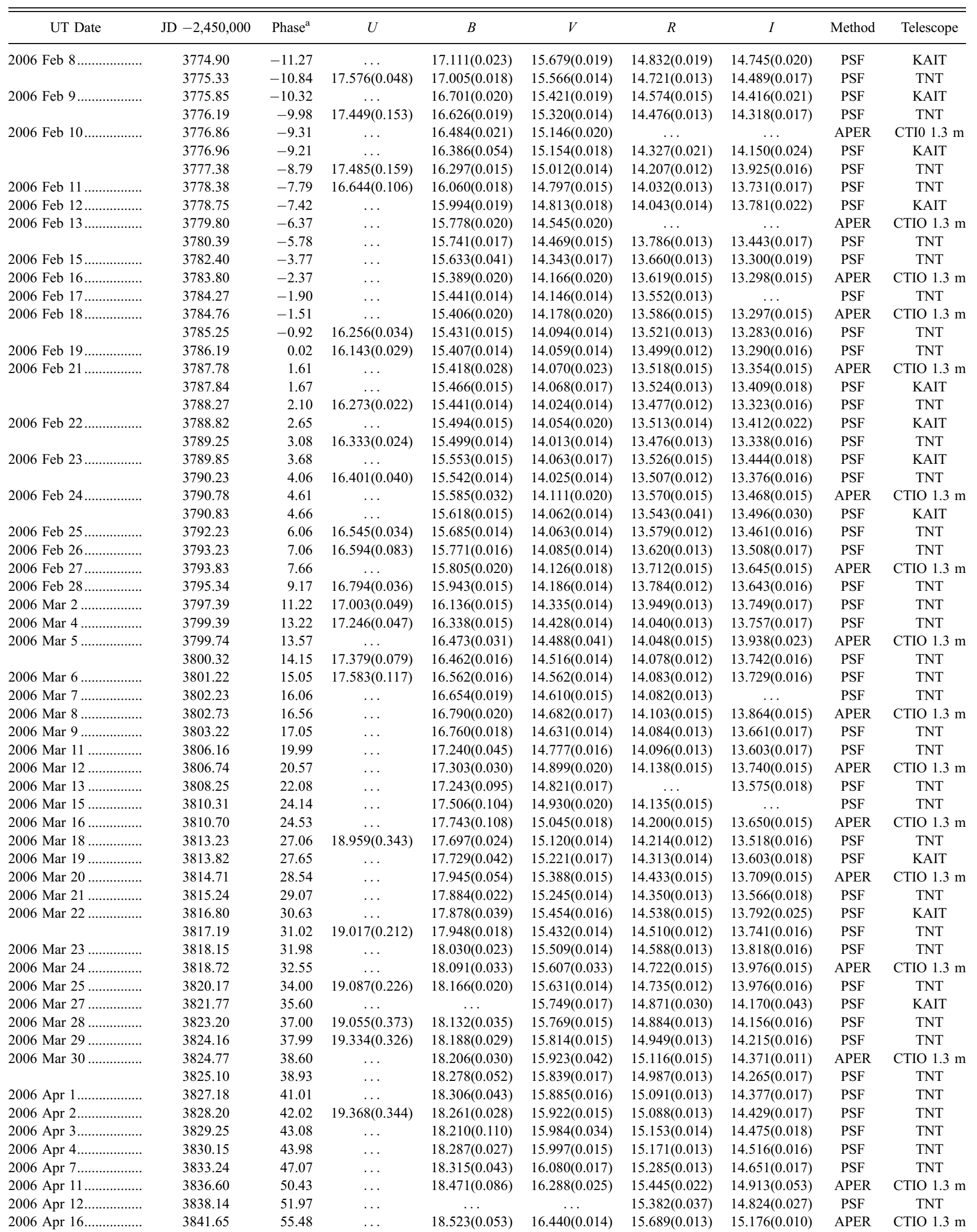


TABLE 3-Continued

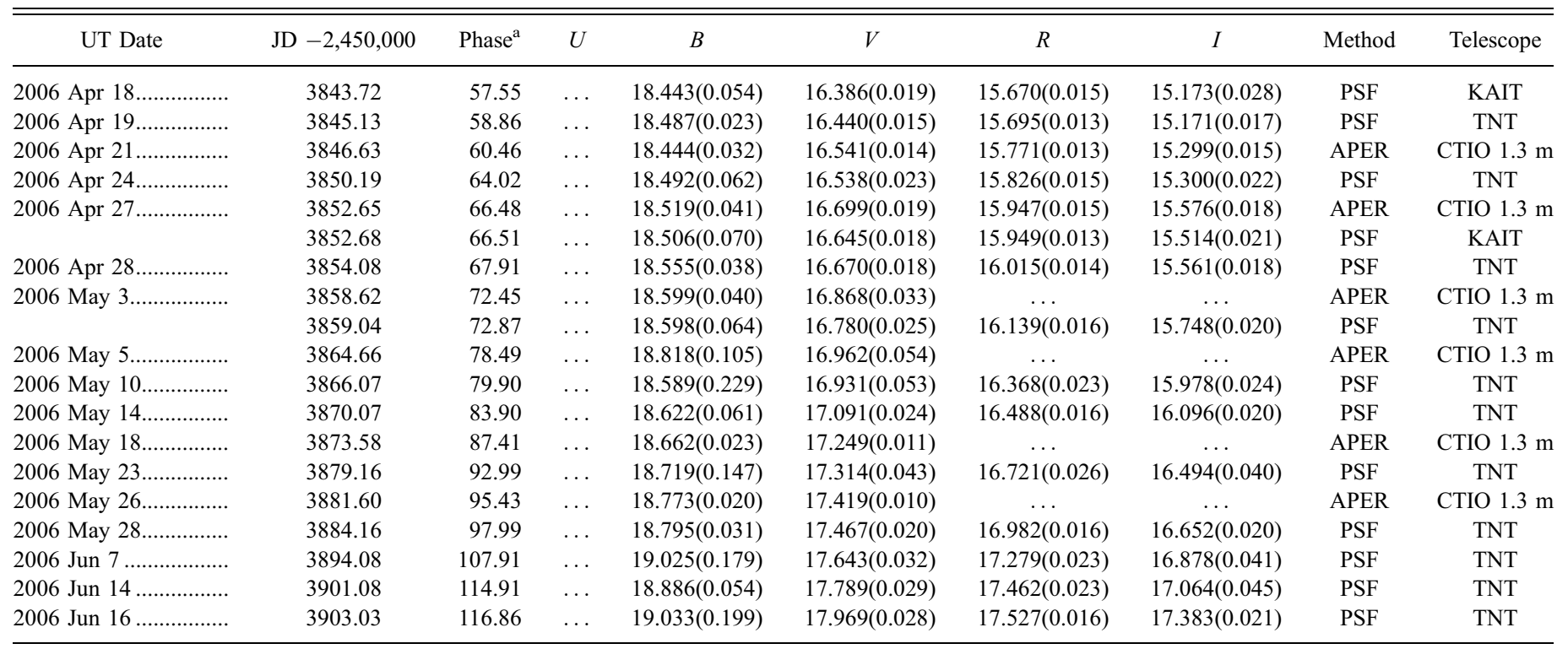

${ }^{\text {a }}$ Relative to the epoch of $B$-band maximum (JD $=2,453,786.17$ ).

$3.0 \mathrm{~m}$ Shane telescope at Lick Observatory using the Kast double spectrograph (Miller \& Stone 1993). Two very late-time spectra were also obtained at the W. M. Keck Observatory: one with the Low Resolution Imaging Spectrometer (LRIS; Oke et al. 1995) mounted on the $10 \mathrm{~m}$ Keck I telescope, and the other with the Deep Extragalactic Imaging Multi-Object Spectrograph (DEIMOS) mounted on the $10 \mathrm{~m}$ Keck II telescope. A journal of spectroscopic observations is given in Table 6 .

All spectra were reduced using standard IRAF routines (e.g., Foley et al. 2003). Extraction of the SN spectra was carefully performed to avoid contamination from the nearby star and the spiral arms of the galaxy. For the Kast observations, flat fields for the red-side spectra were taken at the position of the object to reduce NIR fringing effects. Flux calibration of the spectra was

TABLE 4

Infrared Photometry of SN 2006X

\begin{tabular}{|c|c|c|c|c|}
\hline UT Date & $\mathrm{JD}-2,450,000$ & Phase $^{\mathrm{a}}$ & $J$ & $K$ \\
\hline 2006 Feb $10 \ldots \ldots \ldots$ & 3776.86 & -9.31 & $13.353(0.013)$ & $13.178(0.038)$ \\
\hline 2006 Feb $13 \ldots \ldots . .$. & 3779.80 & -6.37 & $12.969(0.015)$ & $12.885(0.034)$ \\
\hline 2006 Feb $17 \ldots \ldots . .$. & 3783.80 & -2.37 & $12.921(0.015)$ & $12.881(0.030)$ \\
\hline 2006 Feb $18 \ldots \ldots \ldots$ & 3784.76 & -1.41 & $12.957(0.015)$ & $12.854(0.030)$ \\
\hline 2006 Feb $21 \ldots \ldots . .$. & 3787.78 & 1.61 & $13.129(0.013)$ & $12.912(0.045)$ \\
\hline 2006 Feb $24 \ldots \ldots \ldots$ & 3790.78 & 4.61 & $13.360(0.013)$ & $13.061(0.043)$ \\
\hline 2006 Feb $27 \ldots \ldots .$. & 3793.83 & 7.66 & $13.802(0.018)$ & $13.229(0.058)$ \\
\hline 2006 Mar $2 \ldots \ldots \ldots$ & 3796.75 & 10.58 & $14.433(0.019)$ & $13.239(0.040)$ \\
\hline 2006 Mar $5 \ldots \ldots \ldots$ & 3799.74 & 13.57 & $14.785(0.029)$ & \\
\hline 2006 Mar $8 \ldots \ldots \ldots$ & 3802.73 & 16.56 & $14.526(0.017)$ & $13.120(0.026)$ \\
\hline 2006 Mar $12 \ldots \ldots$. & 3806.74 & 20.57 & $14.328(0.017)$ & $12.945(0.039)$ \\
\hline 2006 Mar $16 \ldots \ldots$. & 3810.70 & 24.53 & $14.071(0.016)$ & $12.854(0.034)$ \\
\hline 2006 Mar $20 \ldots \ldots$. & 3814.71 & 28.54 & $13.780(0.016)$ & $12.899(0.051)$ \\
\hline 2006 Mar $24 \ldots \ldots .$. & 3818.72 & 32.55 & $13.909(0.018)$ & $12.933(0.043)$ \\
\hline 2006 Mar $30 \ldots \ldots$. & 3824.77 & 38.60 & $14.480(0.019)$ & $13.648(0.028)$ \\
\hline 2006 Apr $11 \ldots \ldots \ldots$ & 3836.60 & 50.43 & $15.282(0.027)$ & $14.103(0.073)$ \\
\hline 2006 Apr $16 \ldots \ldots \ldots$ & 3841.65 & 55.48 & $15.727(0.034)$ & $14.350(0.043)$ \\
\hline 2006 Apr $21 \ldots \ldots .$. & 3846.63 & 60.46 & $15.943(0.036)$ & $14.569(0.043)$ \\
\hline 2006 Apr $27 \ldots \ldots .$. & 3852.65 & 66.48 & $16.302(0.047)$ & $14.601(0.058)$ \\
\hline 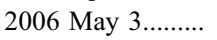 & 3858.62 & 72.45 & $16.521(0.128)$ & \\
\hline 2006 May $9 \ldots \ldots \ldots . .$. & 3864.66 & 78.49 & $17.121(0.096)$ & $15.124(0.072)$ \\
\hline
\end{tabular}

${ }^{\text {a }}$ Relative to the epoch of $B$-band maximum (JD $\left.=2,453,786.17\right)$. performed by means of spectrophotometric standard stars observed at similar air mass on the same night as the SN. The spectra were corrected for continuum atmospheric extinction using mean extinction curves for BAO and Lick Observatory; also telluric lines were removed from the Lick (but not BAO) data. For spectra observed at Lick and Keck, the slit was always aligned along the parallactic angle to avoid chromatic effects in the data (Filippenko 1982). This procedure was not adopted at BAO, however, and it may not significantly affect the continuum shape as the air mass was usually low $(\sim 1.1)$ when getting these spectra for SN 2006X. In general, the relative spectrophotometry is quite good, consistent with the broadband photometry within about $5 \%$.

\section{LIGHT CURVES AND SPECTRA}

\subsection{Optical and Near-IR Light Curves}

The UBVRIJK light curves of SN 2006X are presented in Figure 2. No $K$-corrections have been applied to any of the magnitudes due to the small redshift of SN $2006 X(z=0.0053)$. For all the bands we have excellent temporal coverage, especially around maximum light. In general, the optical measurements obtained with TNT, KAIT, and the CTIO $1.3 \mathrm{~m}$ telescope are consistent with each other within $\pm 0.05 \mathrm{mag}$, except in the $I$ band where differences are more noticeable. We find that the TNT I-band magnitudes are systematically brighter than those of KAIT by $0.08 \pm 0.02 \mathrm{mag}$ and also brighter than those of the CTIO $1.3 \mathrm{~m}$ telescope, especially around the dip immediately after maximum where the discrepancy reaches $\sim 0.2 \mathrm{mag}$. This larger difference is likely caused by the different instrument response function in the $I$ band for the different systems.

Besides the usual color-term corrections, additional magnitude corrections between instrumental and standard bandpasses ( $S$-corrections; Stritzinger et al. 2002) are sometimes required for the filters that are different from those defined by Bessell (1990). The filters mounted on TNT are standard JohnsonCousins $U B V R I$ system; however, the filter transmission curves are unavailable. We obtained the $S$-corrections for observations of the CTIO $1.3 \mathrm{~m}$ telescope (see Table 5), except in $R$ and $I$ bands where the corrections were found to actually increase the systematic differences of the photometry obtained with different telescopes (e.g., Krisciunas et al. 2003). We thus have chosen 
TABLE 5

Optical and NiR $S$-Corrections Added to the Data of the CTIO $1.3 \mathrm{~m}$ Telescope Listed in Tables 3 and 4

\begin{tabular}{|c|c|c|c|c|c|c|}
\hline UT Date & JD $-2,450,000$ & Phase $^{a}$ & $B$ & $V$ & $J$ & $K$ \\
\hline 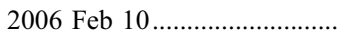 & 3776.86 & -9.31 & -0.046 & -0.004 & 0.038 & -0.003 \\
\hline 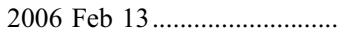 & 3779.80 & -6.37 & -0.043 & 0.001 & 0.046 & 0.005 \\
\hline 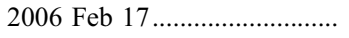 & 3783.80 & -2.37 & -0.043 & 0.010 & 0.059 & 0.019 \\
\hline 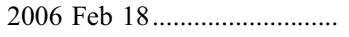 & 3784.76 & -1.41 & -0.044 & 0.012 & 0.062 & 0.022 \\
\hline 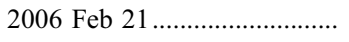 & 3787.78 & 1.61 & -0.044 & 0.017 & 0.067 & 0.034 \\
\hline 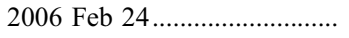 & 3790.78 & 4.61 & -0.043 & 0.021 & 0.036 & 0.057 \\
\hline 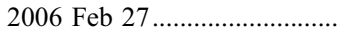 & 3793.83 & 7.66 & -0.039 & 0.023 & 0.007 & 0.054 \\
\hline 2006 Mar 2 ............................. & 3796.75 & 10.58 & $\ldots$ & $\ldots$ & -0.018 & 0.043 \\
\hline 2006 Mar 5 .............................. & 3799.74 & 13.57 & -0.024 & 0.026 & -0.043 & $\ldots$ \\
\hline 2006 Mar 8 ............................ & 3802.73 & 16.56 & -0.014 & 0.027 & -0.066 & 0.018 \\
\hline 2006 Mar 12 ........................... & 3806.74 & 20.57 & -0.003 & 0.029 & -0.091 & 0.004 \\
\hline 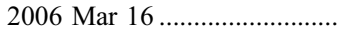 & 3810.70 & 24.53 & -0.019 & 0.032 & -0.076 & -0.010 \\
\hline 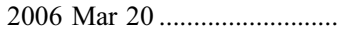 & 3814.71 & 28.54 & -0.066 & 0.036 & -0.057 & -0.016 \\
\hline 2006 Mar 24 ........................... & 3818.72 & 32.55 & -0.069 & 0.039 & -0.09 & -0.007 \\
\hline 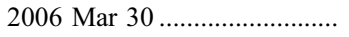 & 3824.77 & 38.60 & -0.066 & 0.041 & -0.119 & -0.005 \\
\hline 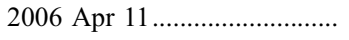 & 3836.60 & 50.43 & -0.056 & 0.030 & -0.131 & 0 \\
\hline 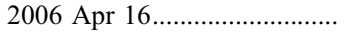 & 3841.65 & 55.48 & -0.049 & 0.025 & -0.131 & 0 \\
\hline 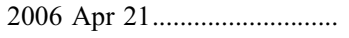 & 3846.63 & 60.46 & -0.041 & 0.026 & -0.131 & 0 \\
\hline 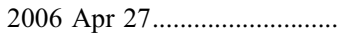 & 3852.65 & 66.48 & -0.032 & 0.026 & -0.131 & 0 \\
\hline 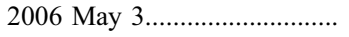 & 3858.62 & 72.45 & -0.032 & 0.025 & -0.131 & 0 \\
\hline 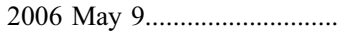 & 3864.66 & 78.49 & -0.032 & 0.025 & -0.131 & 0 \\
\hline 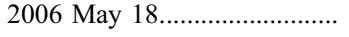 & 3873.58 & 87.41 & -0.032 & 0.025 & $\ldots$ & $\cdots$ \\
\hline 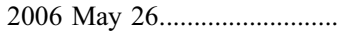 & 3881.60 & 95.43 & -0.032 & 0.025 & $\ldots$ & $\ldots$ \\
\hline
\end{tabular}

${ }^{\text {a }}$ Relative to the epoch of $B$-band maximum (JD $=2,453,786.17$ ).

to let the ANDICAM $R$ and $I$ photometries stay as they are. The $S$-corrections for the filters closer to Bessell's recipe are generally small in $B V R$ (e.g., $\lesssim 0.05 \mathrm{mag}$ ) but probably significantly larger in $U$ and $I$ (e.g., Pastorello et al. 2007). This is demonstrated by the consistency of the raw magnitudes of TNT and KAIT with the $S$-corrected magnitudes of the CTIO $1.3 \mathrm{~m}$ in $B$ and $V$ bands within their errors (see Fig. 2). To account for the I-band magnitude difference between the TNT, KAIT, and the CTIO $1 \mathrm{~m}$ telescope, we assign a systematic error of $0.10 \mathrm{mag}$ for all the $I$-band magnitudes in the following analysis.

A polynomial fit to the $B$-band light curve around maximum yields $B_{\max }=15.40 \pm 0.03 \mathrm{mag}$ on JD 2, 453, 786.17 \pm 0.35 (2006 February 19.93). This means that our observations started from -11.27 days and extended to +116.32 days with respect to the time of $B$ maximum. Likewise, the $V$ light curve reached a peak magnitude of $14.04 \pm 0.02$ on JD $2,453,789.11 \pm 0.29$, about 2.9 days after $B$ maximum. The fitting parameters for the maxima in the other bands are presented in Table 7, together with the late-time decline rates (see below). From the $B$ and $V$ light curves we derived an observed $\Delta m_{15}(B)=1.17 \pm 0.05 \mathrm{mag}^{7}$ and $B_{\max }-V_{\max }=1.36 \pm 0.04$ mag. We also measured the $B-V$ color at 12 days after $B$ maximum to be $1.83 \pm 0.05 \mathrm{mag}$. These colors are much redder than the intrinsic values shown by other SNe Ia with similar $\Delta m_{15}(B)$ (see more discussion in $\S 3.3$ ), suggesting that SN 2006X suffered significant line-of-sight reddening.

7 The true $\Delta m_{15}(B)$ for SN $2006 \mathrm{X}$ is $1.31 \pm 0.05$, taking into account the reddening effect on the light-curve shape (Phillips et al. 1999).

TABLE 6

Journal of Spectroscopic Observations of SN 2006X

\begin{tabular}{|c|c|c|c|c|c|}
\hline UT Date & $\mathrm{JD}-2,450,000$ & $\begin{array}{l}\text { Phase }^{\mathrm{a}} \\
\text { (days) }\end{array}$ & $\begin{array}{l}\text { Range } \\
(\AA)\end{array}$ & $\begin{array}{c}\text { Resolution } \\
(\AA)\end{array}$ & Instrument \\
\hline 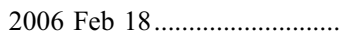 & 3785.3 & -0.9 & $3700-8500$ & 4 & BAO 2.16 m Cassegrain \\
\hline \multirow{2}{*}{ 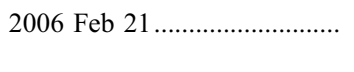 } & 3787.7 & +1.5 & $3300-11000$ & $5-12$ & Lick Shane 3.0 m Kast \\
\hline & 3788.3 & +2.1 & $4000-9000$ & 3 & BAO $2.16 \mathrm{~m}$ BFOSC \\
\hline 2006 Feb 25 & 3792.3 & +6.1 & $3700-8200$ & 4 & BAO $2.16 \mathrm{~m}$ Cassegrain \\
\hline 2006 Mar 4 & 3799.2 & +13.1 & $3700-8200$ & 4 & BAO $2.16 \mathrm{~m}$ Cassegrain \\
\hline $2006 \operatorname{Mar} 21$ & 3816.2 & +30.1 & $4000-9000$ & 3 & BAO 2.16 m BFOSC \\
\hline 2006 Mar 22 & 3816.7 & +30.6 & $3300-11000$ & $5-12$ & Lick Shane 3.0 m Kast \\
\hline 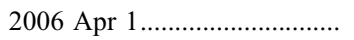 & 3827.2 & +41.0 & $4500-6200$ & 4 & BAO 2.16 m Cassegrain \\
\hline 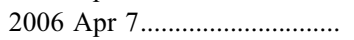 & 3833.3 & +47.1 & $4500-9000$ & 3 & BAO $2.16 \mathrm{~m}$ BFOSC \\
\hline 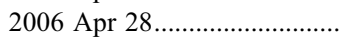 & 3853.9 & +67.7 & $3900-10500$ & $5-12$ & Lick Shane $3.0 \mathrm{~m}$ Kast \\
\hline 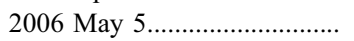 & 3860.9 & +74.7 & $3900-10500$ & $6-12$ & Lick Shane $3.0 \mathrm{~m}$ Kast \\
\hline 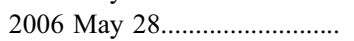 & 3884.0 & +97.8 & $3300-11000$ & $5-11$ & Lick Shane $3.0 \mathrm{~m}$ Kast \\
\hline 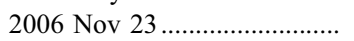 & 4063.2 & +277.0 & $3100-9200$ & 6 & Keck I 10 m LRIS \\
\hline 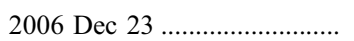 & 4093.2 & +307.0 & $4500-7200$ & 0.35 & Keck II 10 m DEIMOS \\
\hline
\end{tabular}

${ }^{\text {a }}$ Relative to the $B$ maximum ( $\left.\mathrm{JD}=2,453,786.17\right)$.

${ }^{\mathrm{b}}$ Approximate spectral resolution (FWHM intensity). 


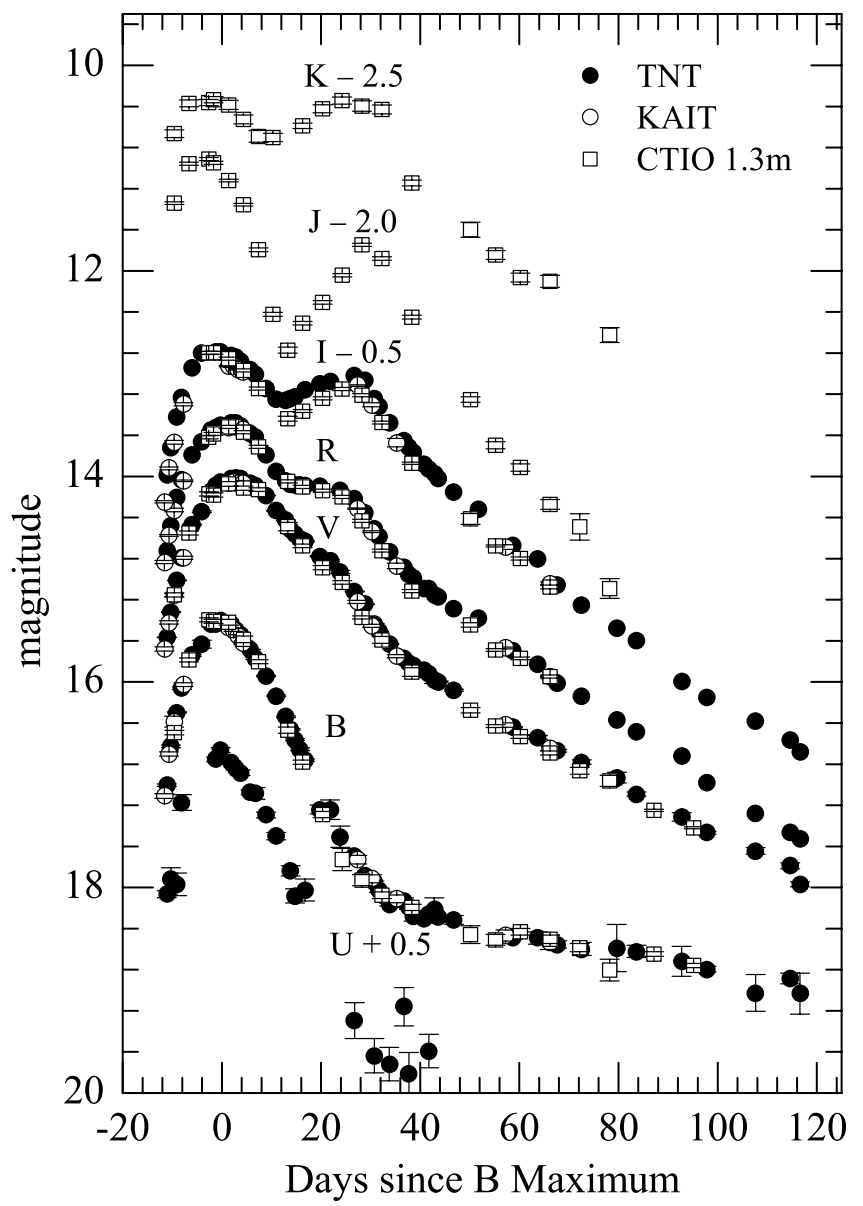

FIG. 2.- UBVRIJK light curves of SN 2006X. Filled circles denote the UBVRI observations from TNT; open circles show the $B V R I$ data from KAIT; open squares represent the $B V R I J K$ data from the CTIO $1.3 \mathrm{~m}$ telescope. The $U, I, J$, and $K$ data have been offset vertically by $+0.5,-0.5,-2.0$, and $-2.5 \mathrm{mag}$, respectively.

In Figures 3-8 we compare the UBVRIJK light curves of SN $2006 X$ with those of other well-observed, nearby SNe Ia having similar $\Delta m_{15}$ values, including SN 1994D $\left(\Delta m_{15}=1.27\right.$; Richmond et al. 1995; Patat et al. 1996), SN 1998bu $\left(\Delta m_{15}=\right.$ 1.04; Jha et al. 1999), SN 2002bo $\left(\Delta m_{15}=1.15\right.$; Benetti et al. 2004; Krisciunas et al. 2004), SN 2002er $\left(\Delta m_{15}=1.32\right.$; Pignata et al. 2004), SN 2003cg $\left(\Delta m_{15}=1.25\right.$; ER06), and SN 2005cf ( $\Delta m_{15}=1.12$; Pastorello et al. 2007). The light curves of SN 1984A $\left(\Delta m_{15}=1.20\right.$; Barbon et al. 1989), although not as well observed as those of the other SNe in the comparison, are also included because SN 1984A shares many similar properties with SN 2006X, especially in the spectroscopy

TABLE 7

Light-Curve Parameters of SN 2006X

\begin{tabular}{|c|c|c|c|}
\hline Band & $t_{\max }$ & $m_{\text {peak }}$ & $\begin{array}{c}\beta^{\mathrm{a}} \\
{\left[\operatorname{mag}(100 \text { days })^{-1}\right]}\end{array}$ \\
\hline$U \ldots \ldots$ & $2453785.13 \pm 0.96$ & $16.20 \pm 0.06$ & $\ldots$ \\
\hline$B \ldots \ldots \ldots \ldots \ldots \ldots \ldots \ldots \ldots \ldots \ldots$ & $2453786.17 \pm 0.35$ & $15.40 \pm 0.03$ & $0.92 \pm 0.05$ \\
\hline$V \ldots \ldots \ldots \ldots \ldots \ldots \ldots \ldots$ & $2453789.11 \pm 0.29$ & $14.04 \pm 0.03$ & $2.71 \pm 0.04$ \\
\hline$R \ldots \ldots \ldots \ldots \ldots \ldots \ldots \ldots \ldots \ldots \ldots \ldots \ldots \ldots$ & $2453789.09 \pm 0.32$ & $13.50 \pm 0.03$ & $3.30 \pm 0.04$ \\
\hline 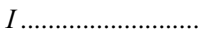 & $2453784.73 \pm 0.40$ & $13.29 \pm 0.10$ & $3.60 \pm 0.02$ \\
\hline$\ldots \ldots \ldots \ldots$ & $2453782.09 \pm 0.38$ & $12.88 \pm 0.02$ & $6.29 \pm 0.24$ \\
\hline ............... & $2453782.97 \pm 0.46$ & $12.82 \pm 0.04$ & $3.78 \pm 0.15$ \\
\hline
\end{tabular}

a The late-time decline rate of the light curve.

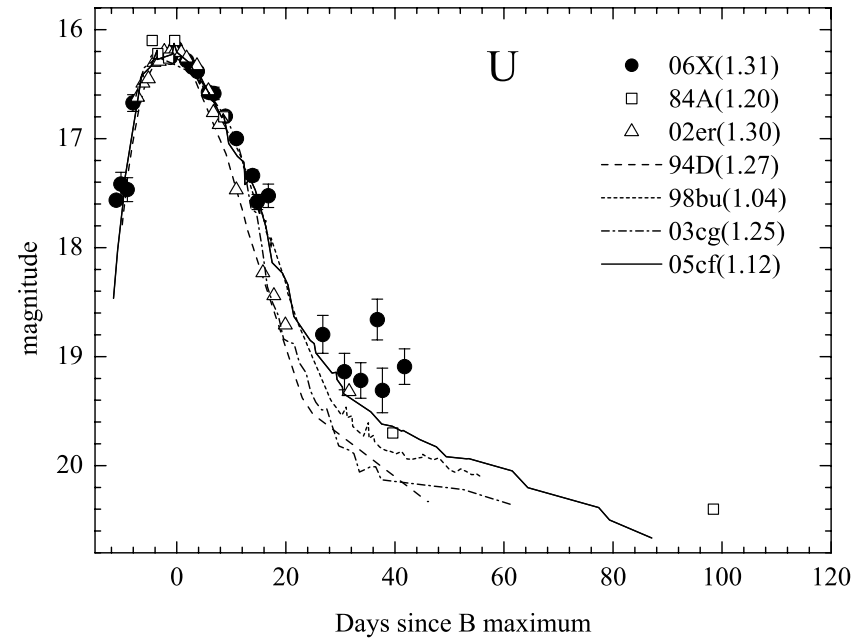

FIG. 3.-Comparison of the observed $U$-band light curves of SN $2006 \mathrm{X}$ with those of SN 1984A, SN 1994D, SN 1998bu, SN 2002er, SN 2003cg, and SN $2005 \mathrm{cf}$. All light curves are arbitrarily shifted in time and magnitude to fit the peak value of SN 2006X. The data sources are cited in the text.

and late-time photometry. All the light curves have been normalized to the epoch of their $B$-band maximum and shifted in peak magnitude to match those of SN 2006X.

Figure 3 shows that the observed $U$-band light curve of $\mathrm{SN}$ $2006 \mathrm{X}$ is slightly broader than those of the other SNe Ia around maximum light but is comparable to that of SN $2003 \mathrm{cg}$, which also suffered significant reddening. Two effects, both associated with large amounts of reddening, might explain why SN $2003 \mathrm{cg}$ and SN 2006X are broader than other SNe in comparison. As demonstrated by Phillips et al. (1999) and Jha et al. (2007), the total-to-selective absorption ratio, $R_{\lambda}$, may vary with the supernova phase. For instance, the true value of $R_{U}$ at 15 days past maximum is found to be smaller than that near maximum by about 0.15 (Jha et al. 2007). This would subsequently broaden the light curves of the reddened SNe by $\sim 0.15 E(B-V)$. On the other hand, the change of the overall spectral shape due to the high reddening may shift the effective bandpass at the shorter wavelengths to the longer wavelengths that are characteristic of broader light-curve peaks (see ER06 for a similar argument for $\mathrm{SN} 2003 \mathrm{cg}$ ). This may also contribute to the broader peak in $U$. Another possible feature of SN 2006X in the $U$ band is the late-time light curve, which, despite the large error bar for each measurement, seems to be brighter and possibly declines more slowly than the other SNe.

Figure 4 shows the comparison in the $B$ band. While the light curves near maximum are similar to each other, they diverge at late times. Compared to other SNe Ia, SN 2006X, SN 1984A, and SN 2002 bo have relatively higher (by $0.3-0.5 \mathrm{mag}$ ) luminosity after $t=40$ days, and their light curves decline more slowly. We measured a late-time decline rate of $\beta=0.92 \pm 0.05 \mathrm{mag}$ (100 days $)^{-1}$ for SN 2006X at $t=40-117$ days, which is comparable to the radioactive decay rate of 0.98 mag $(100 \text { days })^{-1}$ for the $\mathrm{Co} \rightarrow \mathrm{Fe}$ decay. The decline rates for SN 1984A and SN 2002 bo are slightly higher, $\beta=1.14 \pm 0.06$ and $1.17 \pm$ 0.10 mag (100 days $)^{-1}$, respectively. All these decline rates are significantly smaller than the typical value of a normal SN Ia, $\beta=1.40 \pm 0.10 \mathrm{mag}(100 \text { days })^{-1}$, as exhibited by the other $\mathrm{SNe}$ Ia in the comparison.

The $V$-band light-curve comparison is shown in Figure 5. At around maximum, SN 2006X is slightly broader and shows a more prominent "shoulder" feature at $t \approx 3$ weeks than other $\mathrm{SNe}$ Ia. Part of the reason for this may be that, compared to other 


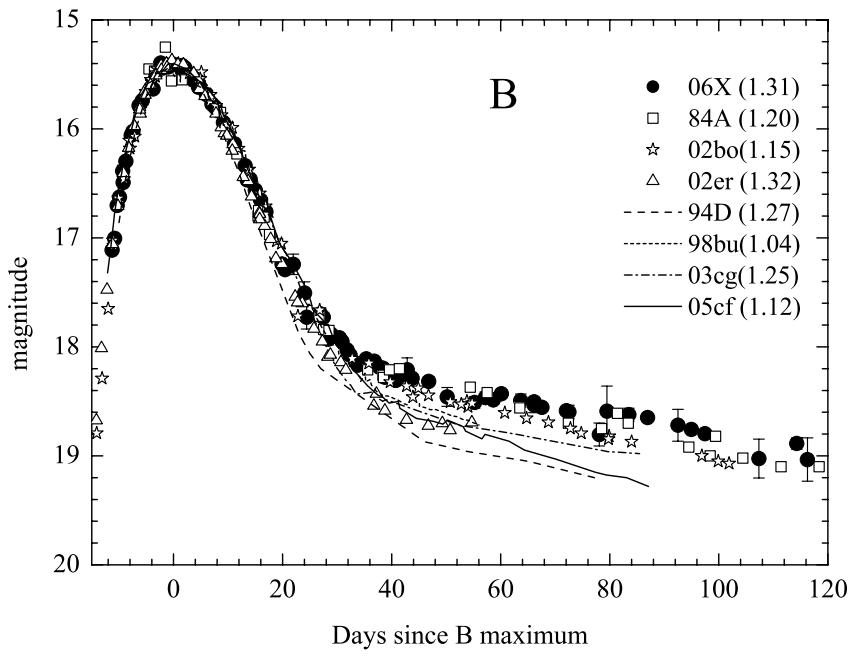

FIG. 4.- Same as Fig. 3, but for the $B$-band light curve.

SNe Ia, SN 2006X has higher reddening, and the effective $V$ bandpass is shifted more toward the $R$ band, which is broader than $V$ and has a prominent shoulder feature. It is also possible that there is an intrinsic scatter in the strength of the shoulder feature in the $V$-band light curves of SNe Ia. In the same manner as the definition of $\Delta m_{15}(B)$, we computed $\Delta m_{15}(V)=0.63 \pm$ $0.03 \mathrm{mag}$ for SN 2006X. This is similar to that of SN $2003 \mathrm{cg}, \mathrm{SN}$ 1998aq, and SN 2005cf $\left[\Delta m_{15}(V)=0.63 \pm 0.05,0.67 \pm 0.05\right.$, and $0.69 \pm 0.03 \mathrm{mag}$, respectively], but smaller than that of SN $1994 \mathrm{D}$ and $\mathrm{SN} 2002 \mathrm{er}\left[\Delta m_{15}(V)=0.85 \pm 0.06\right.$ and $0.78 \pm$ $0.04 \mathrm{mag}$, respectively]. At late times, SN 2006X has a similar evolution as SN 2002bo, and both are brighter than other comparison SNe Ia. There are no obvious differences in the late-time decline rates among the $\mathrm{SNe}$.

As shown in Figures 6 and 7, SN 2006X exhibits a more prominent "plateau" feature in the $R$ band and a stronger secondary maximum in the $I$ band than other SNe Ia. The $R$ and $I$ light curves of SNe Ia are similar to each other before $t=10$ days but diverge after that. There are considerable differences in $D_{\text {plateau }}$, the duration of the plateau phase in the $R$ band, ${ }^{8}$ and the

\footnotetext{
${ }^{8} D_{\text {plateau }}$ measures the interval of the two inflection times, $t_{1}$ and $t_{2}$, of the postmaximum $R$-band light curves. The inflection time is defined as the moment when the first derivative at the transition phase $S=|d m / d t|$ is minimal (e.g., Elmhamdi et al. 2003).
}

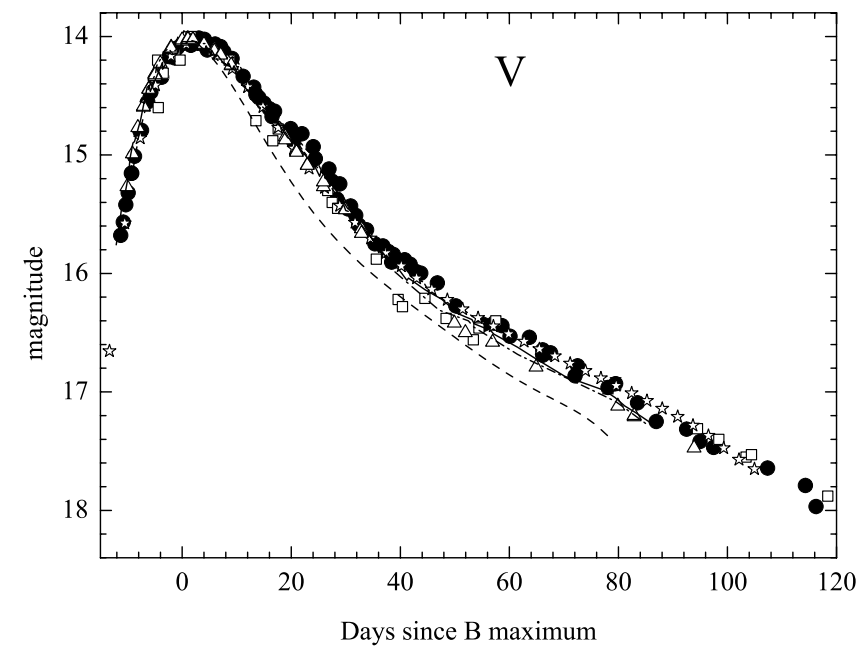

FIG. 5.- Same as Fig. 3, but for the $V$-band light curve.

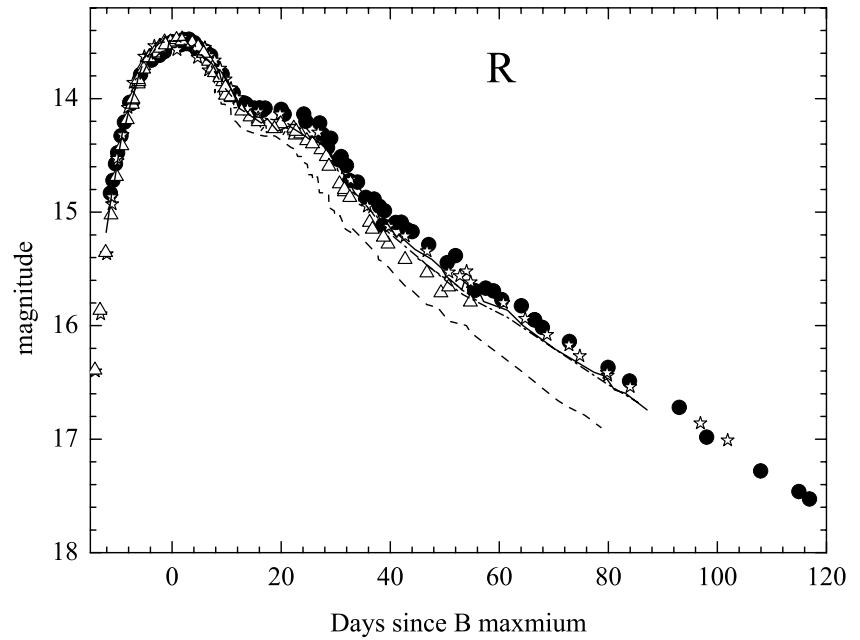

FIg. 6.-Same as Fig. 3, but for the $R$-band light curve.

time when the $I$ band reaches the second maximum $\left[t\left(I_{\max }\right)\right]: \mathrm{SN}$ $2006 \mathrm{X}$ has $D_{\text {plateau }} \approx 13$ days and $t\left(I_{\max }\right)=28$ days, while SN $1994 \mathrm{D}$ has $D_{\text {plateau }} \approx 9$ days and $t\left(I_{\max }\right)=20$ days. At late times, SN 2006X has an evolution similar to that of SN 2002bo in the $R$ band, and they are brighter than other SNe Ia. The two $\mathrm{SNe}$, however, have different decline rates in the I band, and SN $2006 \mathrm{X}$ is the brightest object in the comparison.

In Figure 8, the $J$ - and $K$-band light curves of SN $2006 \mathrm{X}$ are compared with those of SN 1998bu, SN 2002bo, SN 2003cg, and SN 2005cf. The light curves of the well-observed SN $2001 \mathrm{el}\left(\Delta m_{15}=1.10 \pm 0.03 \mathrm{mag}\right.$; Krisciunas et al. 2003$)$ are also overplotted (see the dotted lines). As in the optical bands, the overall light curves of SN 2006X in $J$ and $K$ resemble closely those of SN 2002bo. After the initial peak, both of these $\mathrm{SNe}$ show a stronger secondary maximum feature in the $J K$ bands than other SNe Ia in the comparison. In SN 2001el, the $J$ second-peak feature is somewhat less pronounced; the light curve remains relatively flat from day +11 to +31 . Note that there is marginal evidence for a third maximum (or shoulder) in the NIR light curves of SN 2006X at $\sim 70$ days after $B$ maximum. Kasen (2006) has come up with an explanation for the secondary maximum and has predicted that some $\mathrm{SNe}$ Ia might show a third maximum at $\sim 80$ days. The variation of the secondary maximum of the NIR light curves can be related to the

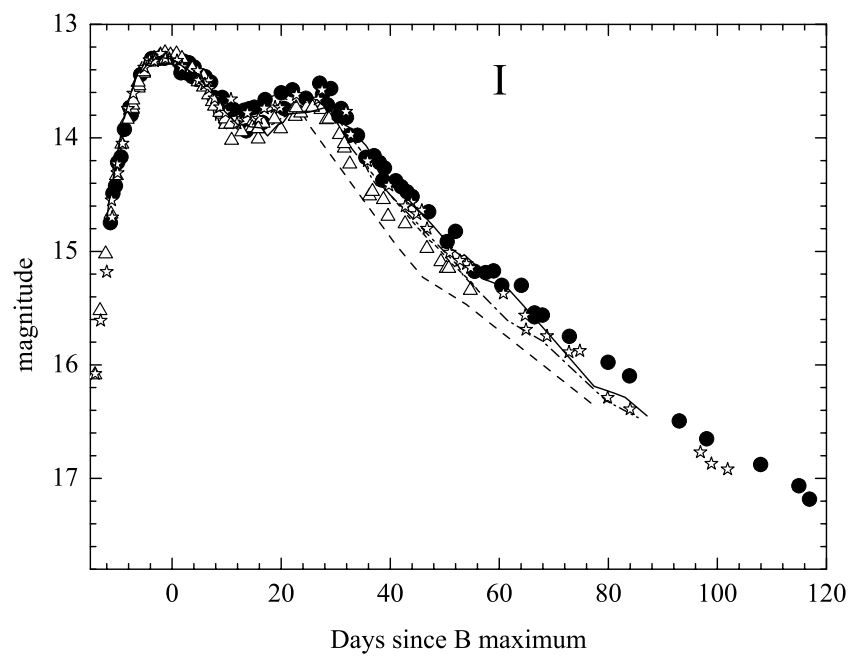

FIG. 7.- Same as Fig. 3, but for the $I$-band light curve. 


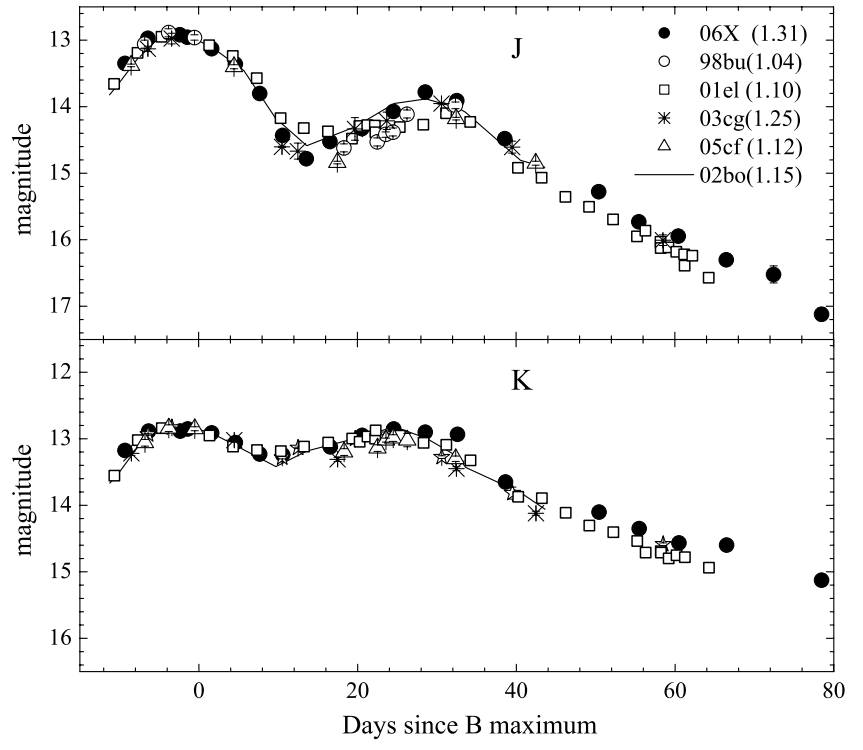

FIG. 8. - NIR $J$ - and $K$-band light curves of SN 2006X compared with those of SN 1998bu, SN 2001el, SN 2002bo, SN 2003cg, and SN 2005cf. The data sources are cited in the text.

abundance stratification in SNe Ia, the concentration of iron group elements, and the progenitor metallicity (Kasen 2006).

\subsection{Optical and Near-IR Color Curves}

The optical color curves of SN 2006X ( $U-B, B-V, V-R$, and $V-I)$ are presented in Figure 9. The colors of SN 2006X are much redder than those of the normal SN Ia SN 2005cf (triangles) with negligible reddening, suggesting a significant amount of reddening toward SN 2006X. Also overplotted in these panels are the color curves of SN 1994D, SN 1998bu, SN 2002bo, SN 2002er, and SN 2003cg, all arbitrarily shifted to match the observed colors of SN 2006X at $B$ maximum.

The $U-B$ color (Fig. 9a) of SN 2006X does not become progressively redder in a linear fashion after $B$ maximum as the other SNe Ia do, but has a shoulder or plateau phase for about 2 weeks. A similar feature is observed in the heavily extinguished $\mathrm{SN} 2003 \mathrm{cg}$. It is unclear whether this feature is caused by the high reddening of these two objects. After $t \approx 4$ weeks, the $U-B$ colors of SN 2006X seem to be bluer than those of the comparison $\mathrm{SNe}$ Ia, but the error bars of the measurements are relatively large due to the poor $U$-band data quality at late times.

The $B-V$ colors of all SNe in the plot (Fig. $9 b$ ) have a similar evolution before $t \approx 29$ days, although SN 2006X reached the bluest color a bit earlier, e.g., at roughly -8.0 days rather than roughly -5 days for the other comparison $\mathrm{SNe}$. All the $\mathrm{SNe} \mathrm{Ia}$ reach the reddest color at $t \approx 29$ days, although there are some differences in the time to reach the reddest color. The peak $B-V$ colors of the $\mathrm{SNe}$ Ia with smaller decline rates (or $\Delta m_{15}$ ) might occur later than those of the faster decliners (see also Fig. 1 in Wang et al. 2005). We note that the $B-V$ color of SN $2003 \mathrm{cg}$ is redder than that of other SNe Ia by about 0.2 mag at the reddest peak according to the data from ER06. However, the photometric data based on the unpublished KAIT data of SN $2003 \mathrm{cg}$ do not have such a discrepancy. Compared with other SNe Ia, the most notable difference in the $B-V$ color evolution of $\mathrm{SN}$ $2006 \mathrm{X}$ is after $t \approx 29$ days: SN 2006X became bluer in a much faster pace and showed an apparent difference from the LiraPhillips relation (the dot-dashed line in the figure; see Lira 1995; Phillips et al. 1999). Note also that the $B-V$ color of SN 1994D, although not well observed during this phase, suggests a faster evolution than the Lira-Phillips relation. Thus, as discussed in the more extreme case of the peculiar SN Ia SN 2000cx ( $\mathrm{Li}$ et al. 2001), although the Lira-Phillips relation provides a method to estimate the extinction toward SNe Ia and may work in a
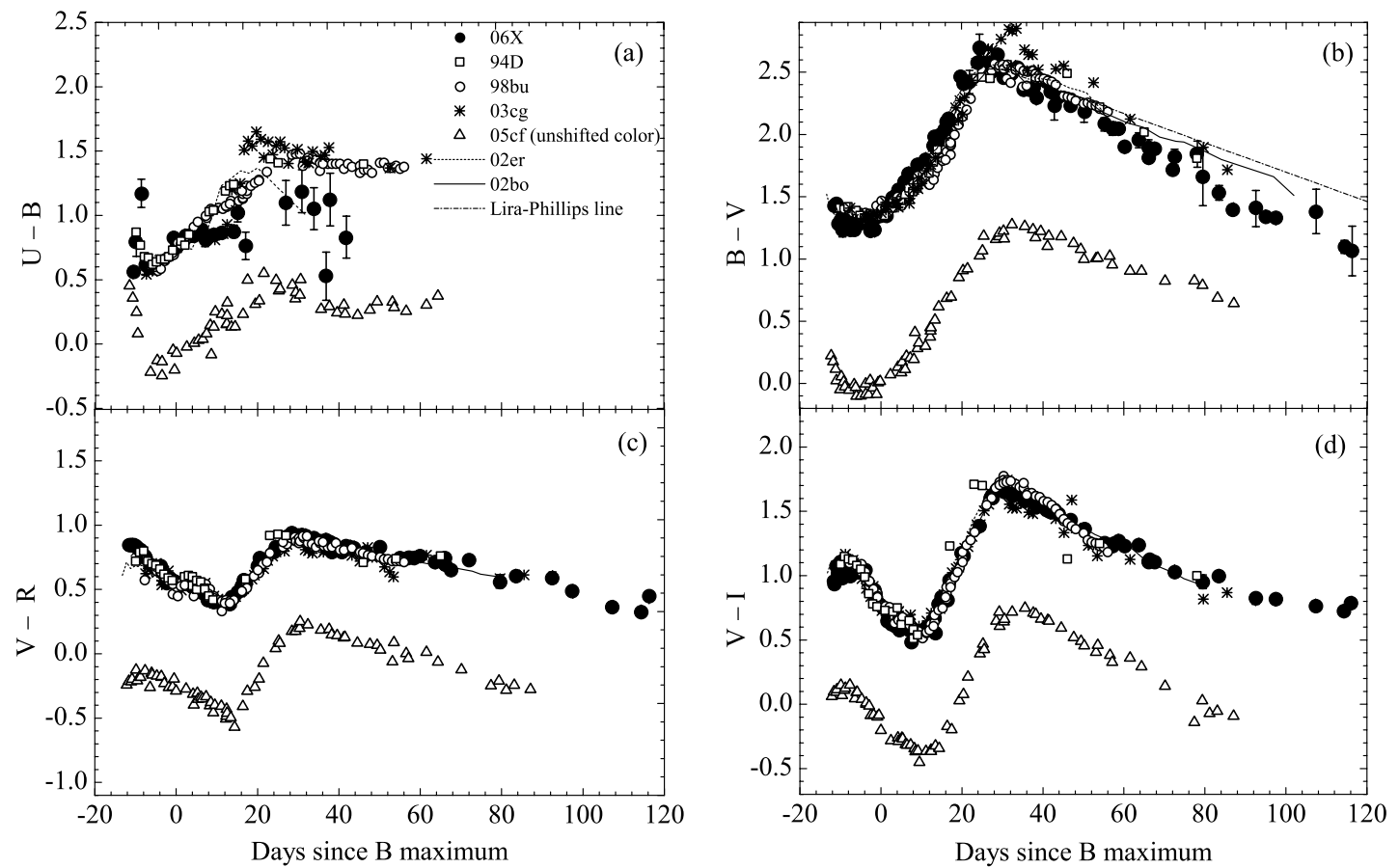

Fig. 9.- $U-B, B-V, V-R$, and $V-I$ color curves of SN 2006X compared with those of SN 1994D, SN 1998bu, SN 2002bo, SN 2002er, SN 2003cg, and SN $2005 \mathrm{cf}$. To show variations in the color evolution between different SNe Ia, the peak colors of all comparison SNe (except for SN $2005 \mathrm{cf}$ ) have been artificially shifted redward to match the observed values of SN 2006X at $B$ maximum. The dot-dashed line shows the Lira-Phillips relation plus a reddening of $E(B-V)=1.42$ mag. The data sources are cited in the text. 


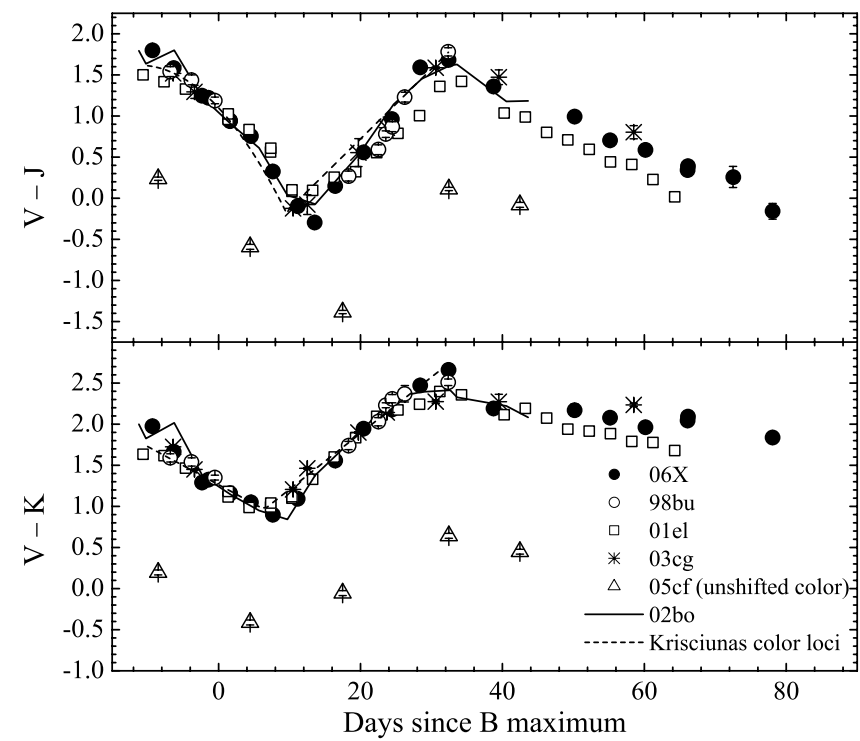

FIG. 10. $-V-J$ and $V-K$ color curves of SN 2006X, together with those of SN 1998bu, SN 2001el, SN 2002bo, SN 2003cg, and SN 2005cf. The dashed lines are the loci of the average light curves from Krisciunas et al. (2000), which are shifted by +1.72 in $V-J$ and +1.95 in $V-K$ in the ordinate direction to minimize the reduced $\chi^{2}$ of the fits.

statistical data set, it should be used with caution for any individual SN Ia.

Given the relatively large differences seen in the $U-B$ and $B-V$ color evolution between SN 2006X and other SNe Ia in the comparison, it is surprising that all $\mathrm{SNe}$ Ia have similar $V-R$ color evolution except at very early times (Fig. 9c). The $V-R$ color of different $\mathrm{SNe}$ Ia reaches the bluest point at $\sim 12$ days after $B$ maximum, then becomes progressively redder and reaches the maximum around day +31 . After the peak the $V-R$ color curve moves gradually toward bluer colors. The similar evolution in the $V-R$ color is related to the closer match of the $V$ and $R$ photometry and also a smaller reddening effect.

The $V-I$ color curve of SN 2006X (Fig. 9d) exhibits a behavior that is very similar to those of the comparison SNe. All these $\mathrm{SNe}$ reached a minimum color at about 9 days after $B$ maximum. The following red peak occurred at around day +31 . The only exception is SN 1994D, which showed a rapid increase in $V-I$ after the minima and then reached the peak value 4 days earlier than other SNe Ia.

Figure 10 shows the observed $V-J$ and $V-K$ colors of SN 2006X, along with those of SN 1998bu, SN 2001el, SN 2002bo, SN 2003cg, and SN 2005cf (without color shift). All SNe Ia exhibit a deep minimum at $t \approx 8-11$ days, followed by a maximum at around day +32 . Nevertheless, the $V-$ NIR color of the comparison SNe Ia shows noticeable scatter, especially in $V-J$ where the magnitude contrast between the valley and peak can differ by more than 0.6 mag. The dashed lines in Figure 10 represent the average loci of the $V-J$ and $V-K$ colors for normal SNe Ia (Krisciunas et al. 2000) that were reddened (shifted upward in Fig. 10) by 1.72 and 1.95 mag, respectively. With respect to the average color curve, the $V-J$ and $V-K$ colors of SN 2002 bo seem to better match those of SN 2006X.

In general, the overall color evolution of SN 2006X resembles most closely those of SN 2002 bo and SN 2002er. Other comparison SNe Ia may match well with SN 2006X in $V-R$ and $V-I$, but they show larger differences than SN 2006X in $B-V$ and $V-$ NIR. Both SN 2006X and SN 2003cg exhibited an atypical "shoulder" feature in $U-B$ for about 2 weeks immediately after $B$ maximum.

\subsection{The Reddening toward SN $2006 X$}

The Galactic extinction toward NGC 4321/SN 2006X is $A_{V}^{\text {gal }}=$ $0.087 \mathrm{mag}$ (Schlegel et al. 1998), corresponding to a color excess of $E(B-V)=0.026$ mag (adopting the standard reddening law of Cardelli et al. 1989). As suggested by the observed color curves of SN 2006X shown in Figures 9 and 10, SN 2006X may suffer a large amount of extinction within its host galaxy. In this section we use two methods, global color curve fitting and the $\Delta m_{15}$ method, to derive the host galaxy reddening for SN 2006X. Both methods assume that SN 2006X has colors similar to those of the other SNe Ia with either similar evolution in some colors or comparable $\Delta m_{15}$ values. Given the somewhat unusual evolution in the $U-B$ (a plateau phase) and $B-V$ (an apparent deviation from the Lira-Phillips relation) colors, however, we caution that SN 2006X may have peculiar intrinsic colors, in which case it would be difficult to estimate the host galaxy reddening toward SN 2006X.

From the comparison of color curves discussed in $\S 3.2$, we find that SN 2006X has a color evolution similar to that of SN 2002 bo in $B-V$ and $V-R, I, J, K$ and to that of SN 2002er in $B-V$ and $V-R, I$. The host galaxy reddenings of SN 2002bo and SN 2002er have been determined by different groups (Reindl et al. 2005; Wang et al. 2006d; Jha et al. 2007) to be $E(B-$ $V)_{\text {host }}=0.40 \pm 0.04$ and $0.16 \pm 0.03 \mathrm{mag}$, respectively. We remove both the Galactic and the host galaxy reddening from SN 2002bo and SN 2002er and create their intrinsic color curves, and then we apply different amounts of color excesses to the colors of SN 2006X and find the best global fit to these intrinsic color curves. We limit the fit to data with $t \lesssim+40$ days since the $B-V$ color of SN $2006 \mathrm{X}$ shows an apparently distinct evolution at late times. This procedure yields the average color excesses $E(B-V)=1.41 \pm 0.07 \mathrm{mag}, E(V-R)=0.61 \pm 0.05 \mathrm{mag}$, $E(V-I)=1.26 \pm 0.05 \mathrm{mag}, E(V-J)=1.76 \pm 0.09 \mathrm{mag}$, and $E(V-K)=1.95 \pm 0.09$ mag.

Phillips et al. (1999) and Wang et al. (2005, 2006d) proposed correlations between the light-curve width parameter $\Delta m_{15}$ and the intrinsic $B_{\max }-V_{\max }$ value (or $C_{\max }$ ) or the $B-V$ color at 12 days after $B$ maximum $\left[(B-V)_{12}\right.$, or $\left.\Delta C_{12}\right]$, which can extend to other optical colors and even to the $V-$ NIR color (see, e.g., Fig. 13 in Krisciunas et al. 2004). We use these two correlations to estimate the host galaxy reddening of SN 2006X. Phillips et al. (1999) found that large reddening changes the observed $\Delta m_{15}$, with $\Delta m_{15}(B)_{\text {true }} \approx \Delta m_{15}(B)_{\text {obs }}+0.1 E(B-V)_{\text {true }}$, where $\Delta m_{15}(B)_{\text {true }}$ and $\Delta m_{15}(B)_{\text {obs }}$ are the intrinsic and observed $\Delta m_{15}(B)$ values, respectively, and $E(B-V)_{\text {true }}$ is the total reddening toward the SN. ER06 suggest that this relation works well for the high-reddening case of SN $2003 \mathrm{cg}$. As we use $\Delta m_{15}(B)_{\text {true }}$ to infer the intrinsic $C_{\max }$ and $\Delta C_{12}$ and derive the reddening toward SN $2006 \mathrm{X}$, this is a loop process; we set $E(B-V)$ as a free parameter and iterate the fitting process until a convergence is achieved.

The color excesses derived from these two methods are consistent with each other within the uncertainties, and they are summarized in Table 8 . The weighted average value of $E(B-V)$ is $1.42 \pm 0.04$ mag, indicating that SN 2006X suffers heavy host galaxy reddening.

Another independent way to estimate the reddening toward SN 2006X is to measure the equivalent width (EW) of the interstellar Na I D doublet, which is proposed to correlate with the amount of line-of-sight dust (Barbon et al. 1990; Munari \& Zwitter 1997). Due to the line blending and the low resolution, 
TABLE 8

Host Galaxy Reddening of SN 2006X Derived from Different Methods

\begin{tabular}{|c|c|c|c|c|c|c|}
\hline Method & $E(U-B)$ & $E(B-V)$ & $E(V-R)$ & $E(V-I)$ & $E(V-J)$ & $E(V-K)$ \\
\hline 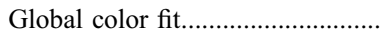 & & $1.41 \pm 0.06$ & $0.61 \pm 0.05$ & $1.27 \pm 0.12$ & $1.76 \pm 0.09$ & $1.95 \pm 0.09$ \\
\hline$C_{\max }$ & $1.23 \pm 0.13$ & $1.38 \pm 0.07$ & $0.54 \pm 0.07$ & $1.02 \pm 0.13$ & $1.71 \pm 0.10$ & $2.04 \pm 0.09$ \\
\hline$C_{12} \ldots \ldots \ldots+\cdots$ & & $1.45 \pm 0.06$ & $0.66 \pm 0.06$ & $1.33 \pm 0.12$ & $1.73 \pm 0.11$ & $1.85 \pm 0.11$ \\
\hline Mean ………… & $1.23 \pm 0.13$ & $1.42 \pm 0.04$ & $0.61 \pm 0.03$ & $1.22 \pm 0.07$ & $1.74 \pm 0.06$ & $1.96 \pm 0.06$ \\
\hline
\end{tabular}

we only use the spectra at days $+13,+68$, and +75 to estimate the $\mathrm{EW}$ of $\mathrm{Na}$ I $\mathrm{D}$. This yields an average $\mathrm{EW} \approx 1.7 \AA$ for $\mathrm{SN}$ 2006X (but see Patat et al. [2007] for measurements of highresolution spectra and the variation of the $\mathrm{Na}$ I D EW). Using the prescription of Barbon et al. (1990), $E(B-V)=0.25 \mathrm{EW}(\mathrm{Na}$ I D), we derive a reddening of $E(B-V) \approx 0.43$ mag toward SN 2006X. However, the prescription by Munari \& Zwitter (1997), with $E(B-V)=0.36 \mathrm{EW}(\mathrm{Na}$ I $\mathrm{D})$, suggests a larger reddening of $E(B-V) \approx 0.61 \mathrm{mag}$. Both determinations are significantly smaller than that derived from the photometric method. Due to the large uncertainty in the correlation between the EW of the $\mathrm{Na}$ I D interstellar absorption and the reddening, we adopt the reddening derived from the photometric method in the following analysis.

\subsection{The Extinction Coefficient $R_{V}$}

Assuming that the dust surrounding SN 2006X conforms with the extinction law proposed by Cardelli et al. (1989), we can set constraints on the value of $R_{V}$. Cardelli et al. (1989) provide an analytic expression for the average extinction law, $A_{\lambda} / A_{V}=a_{\lambda}+$ $b_{\lambda} / R_{V}$ (where $a_{\lambda}$ and $b_{\lambda}$ are wavelength-dependent parameters), which well reproduce the average extinction curve of the Galaxy with $R_{V}=3.1$. The value of $R_{V}$ for SN 2006X can be obtained by simultaneously matching different color excesses derived in $\S 3.3$ using our adopted $E(B-V)$ of $1.42 \pm 0.04 \mathrm{mag}$.

Figure 11 shows that the best fit to the mean color excesses (UBVRIJK minus $V$ ) is achieved with $R_{V}=1.48 \pm 0.06$, which is much smaller than the standard value of 3.1. This gives a host galaxy visual extinction $A_{V}$ (host) $=2.10 \mathrm{mag}$ for SN 2006X. Excluding the color excesses derived from the NIR bands in the fit yields the optimal $R_{V}=1.58 \pm 0.15$ and $A_{V}$ (host) $=2.24 \mathrm{mag}$.

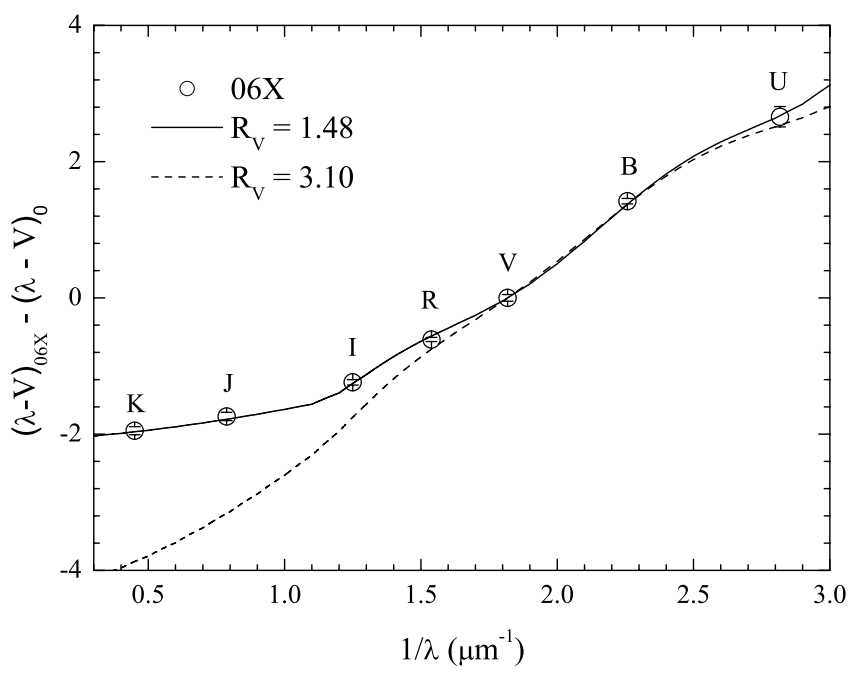

FIG. 11.-UVBRIJK minus $V$ color differences between SN 2006X and the unreddened SNe Ia with similar values of $\Delta m_{15}$. The solid line shows the best-fit extinction curve following the analytical model of Cardelli et al. (1989). The dashed line represents the standard extinction curve with $R_{V}=3.1$.
Allowing the best fit $E(B-V)_{\text {host }}$ to vary marginally alters these results. Fitting the color excesses of SN 2006X using the standard extinction law $\left(R_{V}=3.1\right)$ yields a reduced $\chi^{2}$ of 284.9 and 21.4 for the UBVRIJK and the UBVRI colors, respectively. This indicates that the dust surrounding SN 2006X is quite different from that observed in the Galaxy, probably of a much smaller grain size than that of typical interstellar dust. Note that the derived $R_{V}$ value is just the average value; the true value may actually show small variance with supernova phase. For comparison, we also determined the host galaxy reddening of SN 2006X using the MLCS2k2 method (Jha et al. 2007), which gives $A_{V}$ (host) $=2.21 \mathrm{mag}$ and $R_{V}=1.58$, quite consistent with our determinations with the optical colors.

Besides SN 2006X, we note that other heavily extinguished SNe Ia also tend to suffer reddening by abnormal dust. In Table 9 we list the color excesses and $R_{V}$ derived for several SNe Ia with $E(B-V)_{\text {host }} \gtrsim 0.50$ mag published in the literature. While the $R_{V}$ values of SN 1995E and SN 2000ce are within $2 \sigma$ of 3.1 given their relatively large error bars, the $R_{V}$ values of the other $\mathrm{SNe}$ are considerably smaller than 3.1. It is interesting to investigate whether the low $R_{V}$ derived for these highly extinguished SNe Ia is universal for all SNe Ia or an exception for a few events. Wang et al. (2006d) derived an average $R_{V}=2.32 \pm 0.12$ for Hubble flow SNe Ia. In the MLCS2k2 method, an $R_{V}$ value lower than 3.1 is often required to fit heavily reddened SNe Ia. More SNe Ia with well-observed multicolor light curves will help diagnose the properties of the dust surrounding SNe Ia. We note that although the infrared light curves themselves suffer little from the dust absorption, their color differences depend more sensitively on the ratio $R_{\lambda}$ than the optical colors (as indicated by the difference between the two curves with different $R_{V}$ in Fig. 11), so they are particularly suitable for studying the $R_{V}$ of SNe Ia.

\section{OPTICAL SPECTRA}

Twelve optical spectra of SN 2006X obtained at BAO and Lick Observatory, spanning $t=-0.9$ to +97.8 days, are displayed in Figure 12. Two late-epoch Keck spectra, taken at days +277.0 and +307.0 , are also shown in the plot. The spectral evolution generally follows that of a typical SN Ia near maximum

TABLE 9

Parameters of Highly Extinguished SNe Ia

\begin{tabular}{cccccc}
\hline \hline SN & Host Galaxy & $E(B-V)_{\text {host }}$ & $R_{V}$ & Colors & Source \\
\hline 1995E........ & NGC 4419 & $0.74 \pm 0.05$ & $2.62 \pm 0.28$ & BVI & 1,2 \\
1996ai....... & NGC 5005 & $1.69 \pm 0.10$ & $2.03 \pm 0.12$ & BVI & 1,2 \\
1999cl........ & NGC 4501 & $1.24 \pm 0.07$ & $1.55 \pm 0.08$ & BVRIJHK & 3 \\
2000ce...... & UGC 4195 & $0.60 \pm 0.06$ & $2.70 \pm 0.30$ & UBVRI & 1,2 \\
2003c........ & NGC 3169 & $1.33 \pm 0.11$ & $1.80 \pm 0.19$ & UBVRIJHK & 4 \\
2006X....... & NGC 4321 & $1.42 \pm 0.04$ & $1.48 \pm 0.06$ & UBVRIJK & 5 \\
\hline
\end{tabular}

REFERENCES.- (1) Wang et al. 2006d; (2) Jha et al. 2007; (3) Krisciunas et al. 2006; (4) ER06; (5) this paper. 


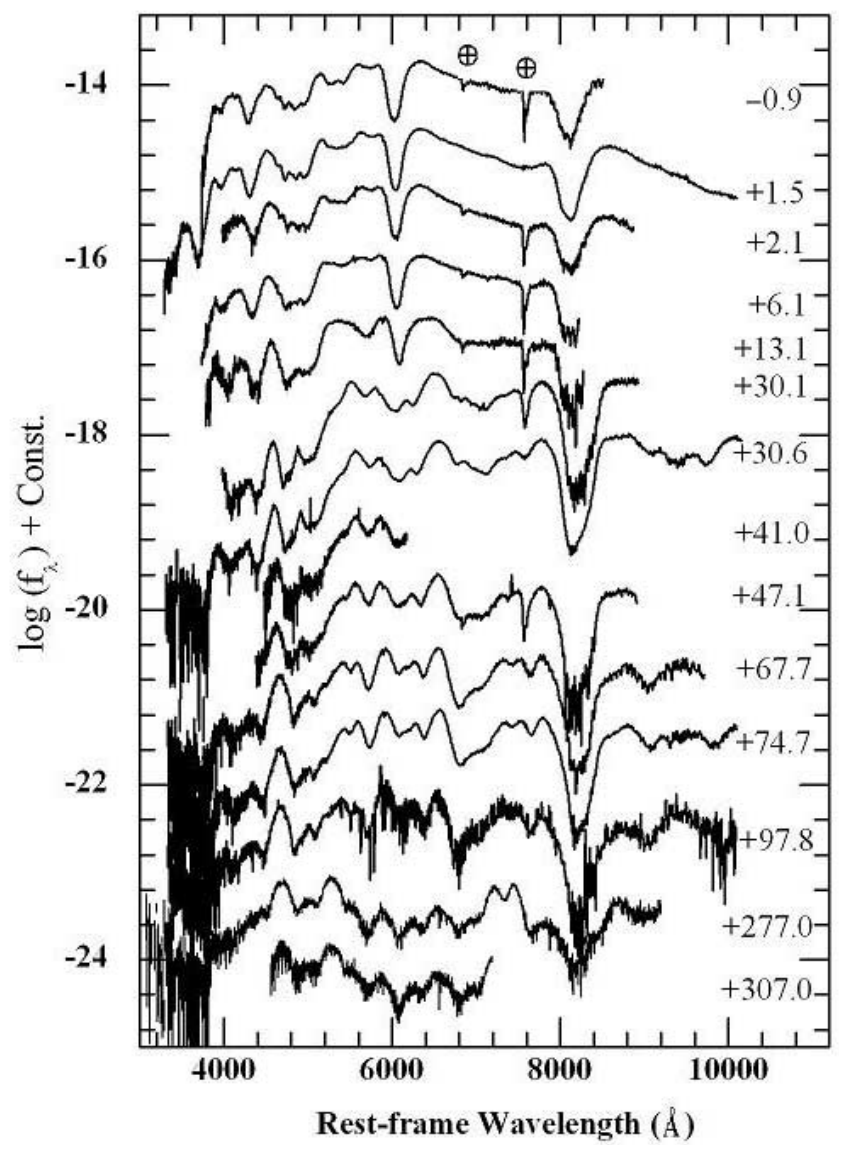

Fig. 12.-Optical spectral evolution of SN 2006X. The spectra have been corrected for the redshift of NGC $4321\left(v_{\text {hel }}=1567 \mathrm{~km} \mathrm{~s}^{-1}\right)$ but not reddening, and they have been shifted vertically by arbitrary amounts for clarity. The numbers on the right-hand side mark the epochs of the spectra in days after $B$ maximum. Uncorrected telluric absorption is marked with a plus sign inside a circle.

brightness, but with more pronounced absorption features at $3700 \AA$ due to $\mathrm{Ca}$ II $\mathrm{H}$ and $\mathrm{K}, 6020 \AA$ due to $\mathrm{Si}$ II $\lambda 6355$, and $8100 \AA$ due to the $\mathrm{Ca}$ II NIR triplet. Other features at early times include the S II lines near $5400 \AA$ and the blended lines of Fe II and Si II near $4500 \AA$. Unlike a normal SN Ia, however, the overall continuum of SN $2006 \mathrm{X}$ appears quite flat within the first 2 weeks after $B$ maximum because of its high reddening. We discuss the detailed spectral evolution of SN 2006X in the following sections.

\subsection{Spectroscopic Evolution at Early Times}

In Figures 13-15, we compare the spectra of SN 2006X with those of SN 1984A (Barbon et al. 1989), SN 1994D (Filippenko 1997), SN 2002bo (Benetti et al. 2004), SN 2002er, and SN $2003 \mathrm{cg}$ (ER06) at several epochs $(t \approx 0$ days, 1 week, and 1 month past $B$ maximum). The spectra of SN 2002er are taken from our unpublished spectral library. All of the spectra have been corrected for reddening and redshift. For SN 2006X, $E(B-V)_{\text {host }}=1.42 \mathrm{mag}$ and $R_{V}=1.48$ are used, while $E(B-$ $V)_{\text {host }}=1.33$ mag and $R_{V}=1.8$ are used for SN $2003 \mathrm{cg}$ (ER06). For the other $\mathrm{SNe}$, the host galaxy reddening is taken from the literature (e.g., Reindl et al. 2005; Wang et al. 2006d; Jha et al. 2007) and extinctions are corrected using the standard extinction law with $R_{V}=3.1$. The line identifications adopted here are taken from Branch et al. (2005, 2006).

At $t \approx 0$ days, the spectra of SN 2006X are characterized by lines of the singly ionized intermediate-mass elements (IMEs):

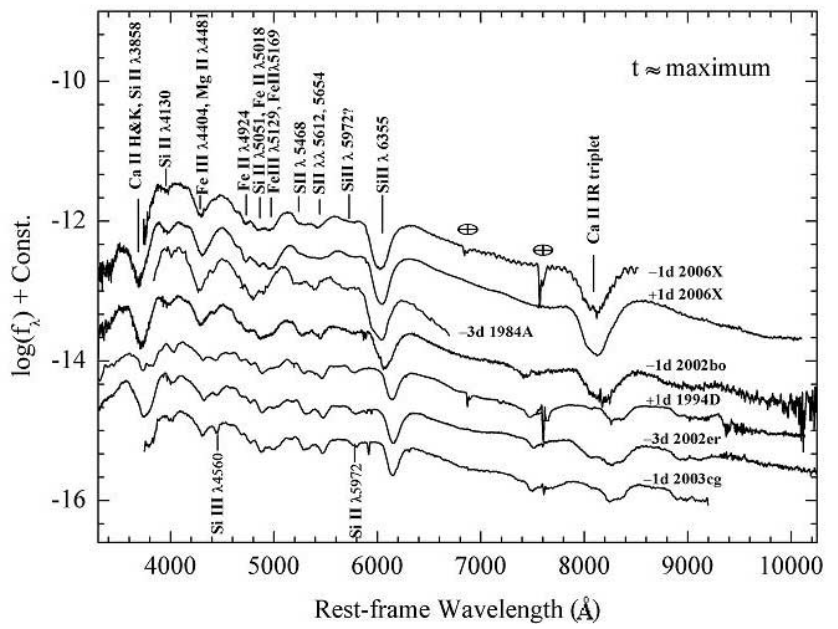

FIG. 13.- Spectrum of SN 2006X near $B$ maximum brightness, overplotted with comparable-phase spectra of SN 1984A, SN 1994D, SN 2002bo, SN 2002er, and SN 2003cg. All spectra shown here and in the subsequent figures have been corrected for reddening and redshift. For clarity of display, the spectra were arbitrarily shifted in the vertical direction.

Ca II, Si II, and Mg II (Fig. 13). The width and strength of these lines are comparable to those seen in SN 1984A and SN 2002bo, but significantly stronger than those of SN 1994D, SN 2002er, and SN $2003 \mathrm{cg}$. The absorption minima due to Si II $\lambda 6355$ is highly blueshifted to $\sim 6020 \AA$ around maximum light, suggesting a high photospheric expansion velocity for SN 2006X. The strong $\mathrm{Ca}$ II $\mathrm{H}$ and $\mathrm{K}$ feature, blended with $\mathrm{Si}$ II 23858 , is more similar to that of SN 2002bo and SN 2002er than to the doubledipped feature seen in SN 1994D and SN 2003cg. The presence or absence of such a double-dipped feature is probably related to the relative strength of $\mathrm{Ca}$ II and $\mathrm{Si}$ II as suggested by Lentz et al. (2000). In the 4000-4500 $\AA$ wavelength range, SN 2006X, SN 1984A, and SN 2002bo share a similar feature with a strong absorption at around $4300 \AA$, probably owing to a blend of $\mathrm{Mg}$ II $\lambda 4481$ and Fe III $\lambda 4404$. The weak feature at $\sim 4400 \AA$ as seen in SN 1994D, SN 2002er, and SN 2003 cg is probably due to the Si III $\lambda \lambda 4553,4568$ blend, which is more sensitive to the temperature in the photosphere. Its absence in the spectra of SN 2006X, SN 2002bo, and SN 1984A suggests a cool temperature. The double S II lines at 5400 and $5600 \AA$ (the W-shaped feature) in SN 2006X are similar to those in the comparison SNe but are less pronounced. The Si II $\lambda 5972$ absorption feature in SN 2006X is very weak, and its strength is difficult to measure. Nevertheless, we measured the potential luminosity indicator parameter $R(\mathrm{Si}$ II $)$ (defined as the line strength ratio of $\mathrm{Si}$ II $\lambda 5972$ to $\mathrm{Si}$ II $\lambda 6355$; Nugent et al. 1995) as $0.12 \pm 0.06$ for SN 2006X, which is much lower than in the other SNe Ia having similar values of $\Delta m_{15}$ (Fig. 3 of Benetti et al. 2005).

At $t \approx 1$ week (Fig. 14), the spectra of all the SNe Ia are still dominated by the Si II, Ca II, and S II lines with increasing contribution from the iron group elements. The W-shaped $\mathrm{S}$ II lines have almost vanished in SN 2006X and SN 1984A, and they appear as an asymmetric absorption trough in SN 2002bo. The absorption at around $4400 \AA$ is probably due to a blend of Fe III $\lambda 4404, \mathrm{Fe}$ II $\lambda 4555$, and $\mathrm{Mg}$ II $\lambda 4481$ and appears at a higher expansion velocity (shorter wavelength) in SN 2002bo, SN 1984A, and SN 2006X than in the other SNe Ia. The Fe II and Si II features in the range 4700-5000 $\AA$ show less substructure in SN 2006X, SN 2002bo, and SN 1984A, while their Si II $\lambda 5051$ line becomes apparently weaker than that of the other comparison $\mathrm{SNe}$ Ia. This probably relates to the high expansion velocity, 


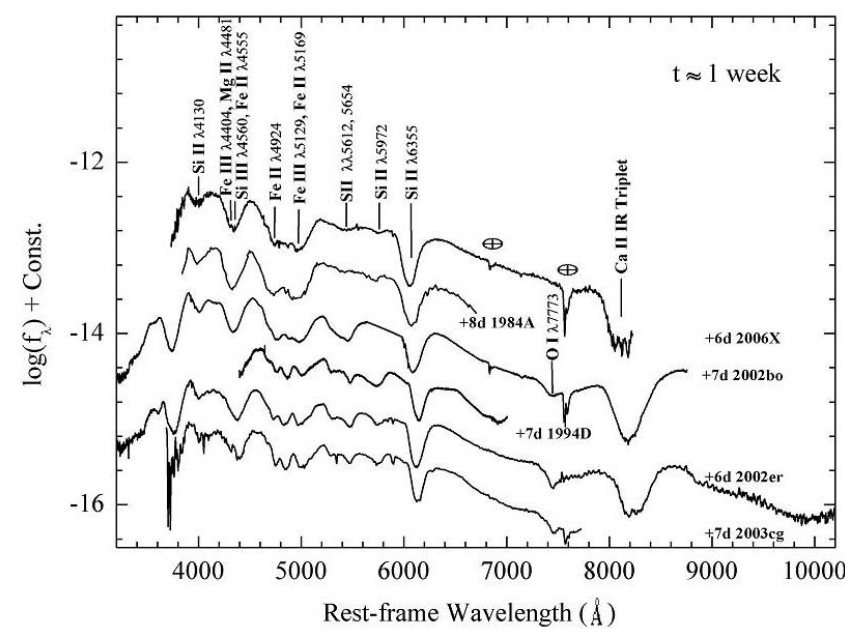

FIG. 14.- Same as Fig. 13, but for spectra about 1 week after $B$ maximum.

which may smear out the weaker features, or it suggests faster evolution of the $\mathrm{S}$ and $\mathrm{Si}$ in the high-velocity SNe Ia. By $t \approx 1$ week the $\mathrm{O}_{\text {I }} 27773$ line strengthens in other SNe Ia but is still rather weak in SN 2006X.

At $t \approx 1$ month (Fig. 15), numerous Fe II lines appear in the spectra of SNe Ia. The Na I D and Si II $\lambda 5972$ blend is progressively stronger. The Si II $\lambda 6355$ trough becomes severely affected by the Fe II $\lambda \lambda 6238,6248$ and Fe II $\lambda \lambda 6456,6518$ lines and is barely visible in SN 2006X and SN 1984A, but it is still present in the spectra of the other comparison $\mathrm{SNe}$ (see the small dip around $6150 \AA$ in Fig. 15). The Ca II NIR triplet feature is stronger and appears at a higher expansion velocity in SN 2002bo and SN 2006X than in the other SNe Ia. The O I line is comparable to that seen in other SNe Ia. It is impressive that the overall appearance and evolution of the spectra of SN 2006X are extremely similar to those of SN 1984A and SN 2002bo at all three epochs (although the spectra of SN 1984A have a limited spectral range). Nevertheless, it is worth pointing out that by $t \approx 1$ month the spectral flux at $\lambda<4000 \AA$ appears relatively higher than that of the comparison $\mathrm{SNe}$ Ia, although the spectrum at this region had a poorer signal-to-noise ratio. The higher spectral flux below $\lambda \sim 4000 \AA$ seems to be also consistent with the bluer $U-B$ color inferred from the photometry at similar phase. The spectrum near maximum light does not

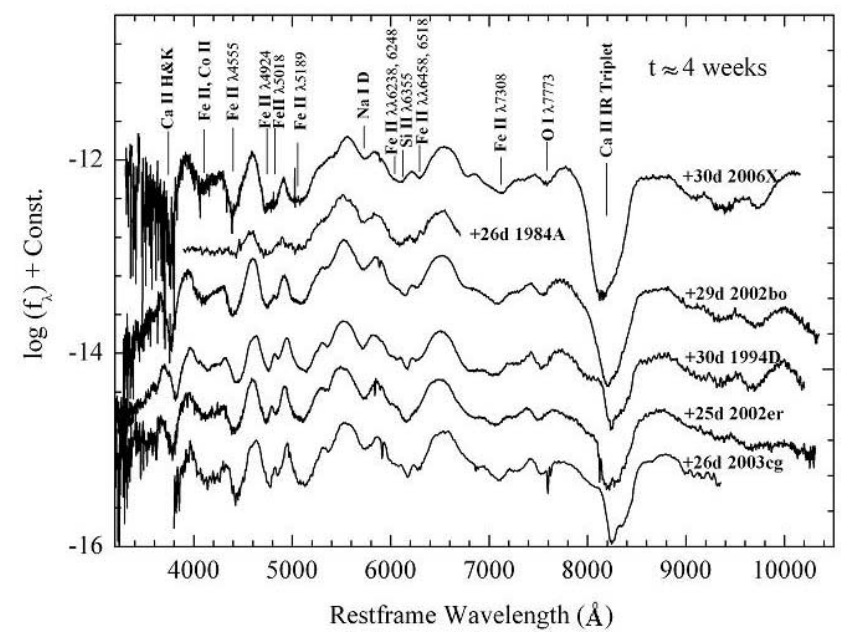

FIg. 15.- Same as Fig. 13, but for spectra at about 4 weeks past $B$ maximum.

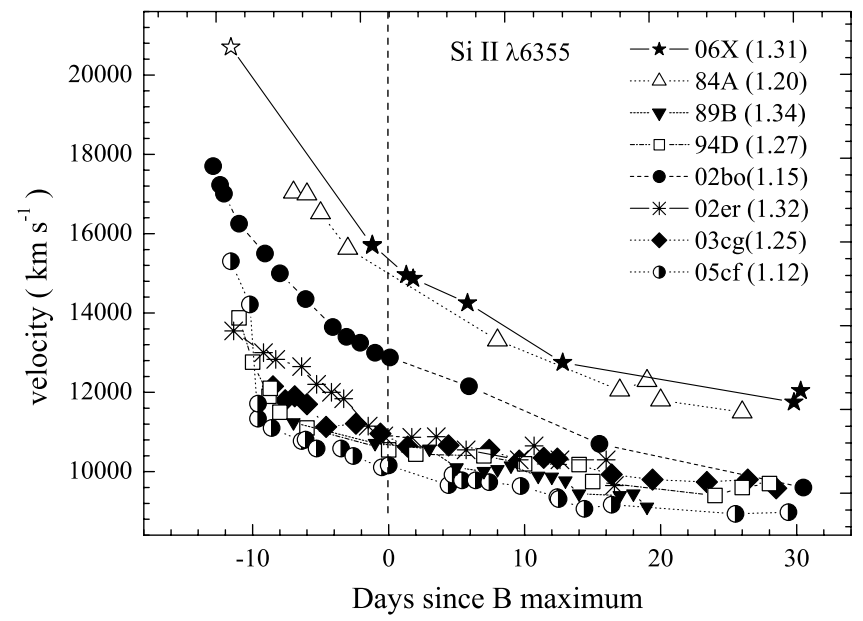

FIG. 16.-Evolution of the expansion velocity of SN 2006X as measured from the minimum of $\mathrm{Si}$ II $\lambda 6355$, compared with the values of SN 1984A taken from Barbon et al. (1989), SN 1989B from Barbon et al. (1990), SN 2002bo from Benetti et al. (2004), SN 1994D from Patat et al. (1996), SN 2003cg from ER06, SN 2002er from Pignata et al. (2004), and SN 2005cf from Garavini et al. (2007). Some of the data points in the plot are from our unpublished spectral library. The numbers in parentheses represent the $\Delta m_{15}$ values for the SNe Ia.

show such a near-UV energy excess. It is unclear when this variation started to occur in SN 2006X, as our spectra taken during the first month past maximum light did not cover the near-UV wavelengths.

\subsection{Expansion Velocity of the Ejecta}

As the photosphere recedes into deeper and more slowly moving ejecta, the P Cygni absorption minima of the spectral features in an SN Ia spectrum gradually shifts redward with time (see also Fig. 12). Thus, the location of the blueshifted minima can in principle trace the photospheric velocity. Indeed, the photospheric velocity $\left(v_{\exp }\right)$ is best measured by the weakest lines, e.g., S II $\lambda 5640$ (Jeffery \& Branch 1990; Mazzali et al. 1993; Patat et al. 1996), although it is usually difficult to measure these weak lines due to blending. The velocities inferred from the strong lines (e.g., Si II 26355 ) represent approximately the $v_{\text {exp }}$ at early phase when the amount of the material above the photosphere is small, but they may not properly indicate the real $v_{\exp }$ as the photosphere falls in the regions where the strong lines could form over a large velocity range due to a flatter density gradient. On account of rather weak S II lines after maximum in SN 2006X, we only examined the velocity evolution of Si II 26355 and the Fe II/ Fe III lines.

The derived $v_{\exp }$ values from Si II $\lambda 6355$ of SN 2006X as a function of time are shown in Figure 16, together with those of the comparison SNe Ia. The $v_{\text {exp }}$ reported for an early-epoch spectrum, taken on February 8.35 (corresponding to $t \approx-11.3$ days) by Quimby et al. (2006), is also included in the plot (shown as the open star). All velocities have been corrected for the redshifts of their respective host galaxies. As can be seen, SN 2006X shows the highest expansion velocities among all $\mathrm{SNe}$ in the comparison, with $v_{\exp }=20,700 \mathrm{~km} \mathrm{~s}^{-1}$ at $t=-11.3$ days and $v_{\exp }=15,700 \mathrm{~km} \mathrm{~s}^{-1}$ at $t=-0.9$ days, while the typical value for most SNe Ia is around $11,000 \mathrm{~km} \mathrm{~s}^{-1}$ at maximum brightness (Filippenko 1997; see also Fig. 1 in Benetti et al. 2005). The $v_{\exp }$ measured from $\mathrm{Ca}$ II $\mathrm{H}$ and $\mathrm{K}$ and $\mathrm{S}$ II 25640 near maximum are about 20,500 and $11,800 \mathrm{~km} \mathrm{~s}^{-1}$, respectively, which are also significantly higher than those measured for the other SNe Ia. SN 1984A and SN 2002bo showed similar, but less pronounced, high-velocity features of the IMEs ( $\mathrm{Si}$ II, S II, and Ca II). Following 


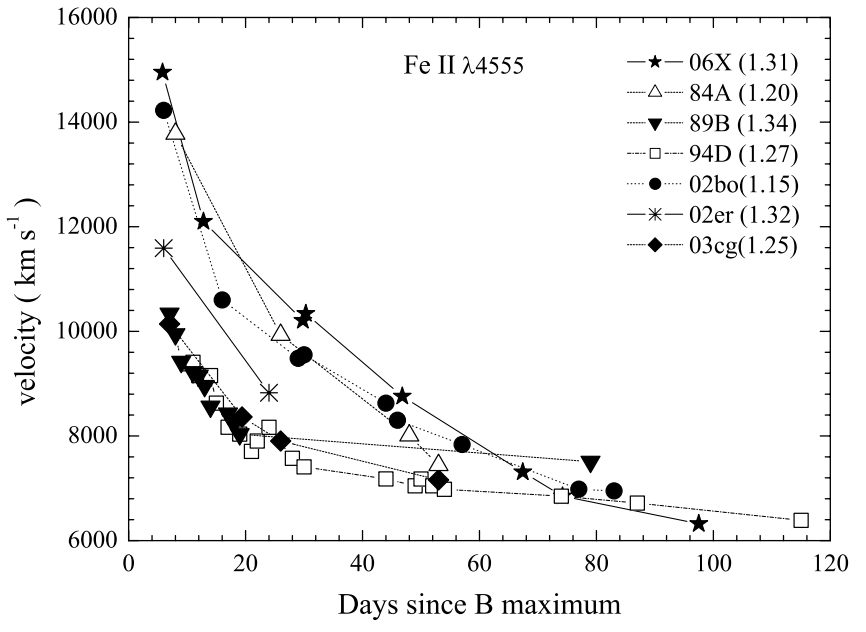

FIG. 17.- Same as Fig. 16, but for the Fe II $\lambda 4555$ line.

Benetti et al. (2005), we calculate the velocity gradient $i$ of Si II $\lambda 6355$ for SN $2006 \mathrm{X}$ during the period from $t \approx 0$ to $t \approx 30$ days as $123 \pm 10 \mathrm{~km} \mathrm{~s}^{-1} \mathrm{day}^{-1}$, which puts SN 2006X in the group of normal SNe Ia with high-velocity gradients (HVGs). Other SNe Ia in the HVG group include SN 1984A and SN 2002bo discussed in this paper, SN 1983G, SN 1989A, SN 1997bp, and SN 2002dj (Benetti et al. 2005), and SN 2004dt (Wang et al. 2006c).

The Fe II and Fe III lines are sometimes used to measure the expansion velocities of the inner ejecta of Fe, providing additional clues to the nature of SN Ia explosions (e.g., Li et al. 2001). The values of $v_{\text {exp }}$ determined from Fe II $\lambda 4555$ and/or Fe III $\lambda 4404$ lines of different SNe Ia are plotted in Figure 17. At early phases the high-excitation Fe III $\lambda 4404$ line is possibly contaminated by $\mathrm{Mg}$ II $\lambda 4481$ and hence may not give a reliable measurement of the $v_{\text {exp }}$. By $t \approx 2-3$ weeks, the Fe II $\lambda 4555$ line becomes stronger and gradually dominates the absorption feature near 4300-4400 $\AA$. This absorption feature yields $v_{\exp } \approx$ $10,000 \mathrm{~km} \mathrm{~s}^{-1}$ for SN $2006 \mathrm{X}$ at $t \approx 1$ month, which is about $3000 \mathrm{~km} \mathrm{~s}^{-1}$ higher than that for SN 1989B and SN 1994D and comparable to that for SN 2000cx ( Li et al. 2001). Likewise, the Fe II lines of SN 1984A and SN 2002bo display high-velocity behavior. As with Si II 26355 , the Fe II feature in these highvelocity events shows more rapid evolution, with larger velocity gradients than in other comparison $\mathrm{SNe}$ Ia. The values of $v_{\text {exp }}$ become much closer to each other when SNe Ia start entering the nebular phase.

The origin of the high-velocity features seen in these SNe Ia is hotly debated. Lentz et al. (2000) find that the strength, profile, and velocity of Si II $\lambda 6355$ are functions of metallicity. The blueward shift of the $\mathrm{Si}$ II feature is found to increase with higher metallicity. However, Benetti et al. (2004) found that increasing the normal metallicity in the $\mathrm{C}+\mathrm{O}$ layer by a factor of 10 in the canonical deflagration model $\mathrm{W} 7$ is not adequate for explaining the large $v_{\exp }$ seen in SN 2002bo. It was also proposed that the high $v_{\text {exp }}$ in these SNe Ia may result from delayed detonation explosions with the transition density from a deflagration to a detonation as the controlling parameter for the internal dispersion of $v_{\exp }$ (Lentz et al. 2001; Benetti et al. 2004). With detailed non-LTE calculations, Lentz et al. (2001) find that some delayed detonation models provide reasonable approximations to the very high velocity feature of SN 1984A. The increase in density of IMEs at higher velocities is thus responsible for the larger blueshifts of the line minimum in the spectra and hence the higher measured expansion velocities.

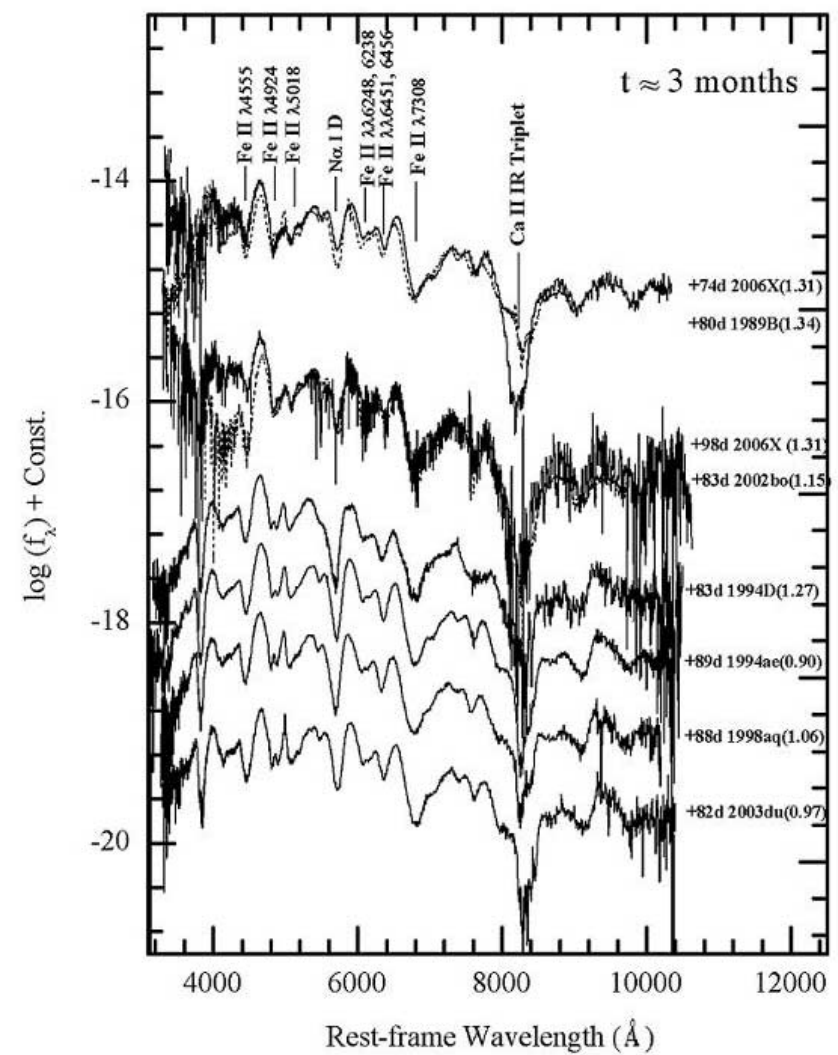

FIG. 18. - Early-epoch nebular spectra of SN 2006X compared with those of other SNe Ia at 3 months past $B$ maximum. The dashed spectra overlapping the spectra of SN 2006X at days +75 and +98 are those of SN 1989B and SN 2002bo at similar epochs. The comparison spectra are from our unpublished spectral library. All the spectra have been vertically shifted for clarity. The numbers in parentheses represent the $\Delta m_{15}$ values for the SNe Ia.

\subsection{Spectra in the Nebular Phase}

Since the $B$-band and probably $V$-band light curves of SN 2006X declined more slowly at late times than those of the other comparison $\mathrm{SNe} \mathrm{Ia}$, it is necessary to examine its late-time spectral behavior in detail. Our collection of nebular-phase spectra of SN 2006X is presented in Figures 18 and 19, together with the spectra of some comparison SNe Ia at similar epochs. Proper corrections for the reddening and redshift have been applied to all the spectra.

In the early nebular phase, at $t \approx 3$ months, the spectra in Figure 18 are quite similar to each other but have subtle differences. The spectra are dominated by various iron lines and have similar relative strengths. The Ca II NIR triplet is still the strongest feature in the earlier nebular spectra. Although the late-time spectra of SN 2006X are rather noisy, they do show a relatively higher flux at $\lambda<5000 \AA$ and an overall bluer continuum than the comparison SNe. The most contrast is with SN 2002bo, in which the relative luminosity in $U$ seems to be fainter than that of SN 2006X by about 1.0 mag. The near-UV excess of SN 2006X might have also occurred in the spectrum at $t \approx 30$ days since $B$ maximum (see Fig. 15). This is consistent with the bluer $U-B$ and $B-V$ colors of SN 2006X compared with the other $\mathrm{SNe}$ (Fig. 9).

Two very late-phase nebular spectra, obtained with the Keck $10 \mathrm{~m}$ telescopes at days +277.0 and +307.0 , are shown in Figure 19. The comparison spectra of SN 1996X and SN 1998bu are from the Suspect online supernova spectrum archive ${ }^{9}$ (contributed

\footnotetext{
9 See http://bruford.nhn.ou.edu/ suspect.
} 


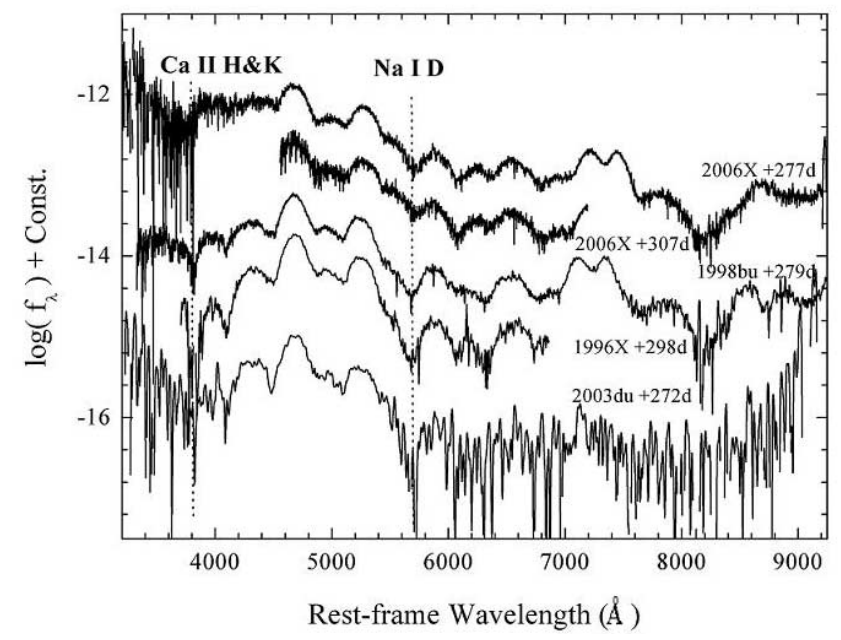

FIG. 19. - Very late-time nebular spectrum of SN 2006X compared with those of SN 1996X, SN 1998bu, and SN 2003du. The two vertical dotted lines refer to the $\mathrm{Ca}$ II $\lambda 3945$ and $\mathrm{Na}$ I $\lambda 5892$ absorptions.

by Salvo et al. 2001; Jha et al. 1999), and the spectra of SN 2003du are from Stanishev et al. (2007). The spectra at this time are dominated by forbidden lines of singly and doubly ionized $\mathrm{Fe}$ and Co. The overall shape of the spectrum of SN 2006X looks similar to that of SN $1998 \mathrm{bu}$, but the profiles and intensities of some features do show significant differences. The most pronounced one is the absorption feature at about $6100 \AA$, which is stronger in SN 2006X than in the other SNe Ia in our comparison. Other discrepancies include the absorption features near 4200 and $4500 \AA$, which are prominent in SN 1996X, SN 1998bu, and SN 2003du but are marginally visible in SN 2006X. We note that both SN 2006X and SN 1998bu show relatively higher flux below $4500 \AA$ at this phase. The extra flux for SN 1998bu could be caused by a light echo (Cappellaro et al. 2001; Spyromilio et al. 2004), which primarily contributes to the spectrum in the blue.

A possible explanation for the abnormal behavior of the nebular-phase spectra and light curves of SN 2006X in the blue is the presence of a light echo. As this supernova is found to suffer significant extinction, it may have occurred in an environment that is abundant with dust, so the occurrence of a light echo is potentially expected. Some of the observable effects of a light echo around an SN Ia include a bluer late-time color, broader spectral lines, and a brighter tail luminosity (Patat 2006), all of which agree with the observations of SN 2006X. The unidentified, prominent feature at $\sim 6100 \AA$ seen in the spectrum at day +307 could be reminiscent of the earlier epoch Si II $\lambda 6355$, reflected by the surrounding dust. Analysis of the light echo in SN 2006X is given in a separate paper (Wang et al. 2008), in which evidence from the late-time HST image and spectrum is presented. In particular, the PSF-subtracted HST image distinctly reveals prominent echo structure at $\sim 0.05^{\prime \prime}-0.13^{\prime \prime}$ away from the SN. Echo emission may also be inferred at $<0.05^{\prime \prime}$ from the center of the residual image (at $\sim 2 \sigma$ level); however, the exact location is unknown due to the limitation of the image resolu- tion. With the Cepheid distance to M100 15.2 Mpc, the materials illuminated by the light echo are found to lie from $<27$ to $\sim 170$ pc from the SN, and it is not clear whether the inner echo was produced from a distinct dust component, e.g., the local circumstellar dust, or from a continuous dust distribution as with the outer echo component.

\section{THE DISTANCE AND LUMINOSITY OF SN 2006X}

The host galaxy of SN 2006X, NGC 4321 (M100), is a wellstudied LINER/H II galaxy (e.g., Ho et al. 1997). As one of the largest spiral galaxies in the Virgo Cluster, it produced SN 1901B, SN 1914A, SN 1959E, SN 1979C, and SN 2006X in roughly the last century. Ferarrese et al. (1997) reported a Cepheid distance to NGC 4321 of $m-M=31.04 \pm 0.17 \mathrm{mag}$. A distance of $m-$ $M=30.91 \pm 0.14$ mag was published by the HST Key Project (Freedman et al. 2001), which we adopt here in our analysis.

\subsection{Absolute Magnitudes and $H_{0}$}

With the Cepheid distance and the reddening derived in the previous sections, it is straightforward to calculate the absolute magnitudes of SN 2006X. After correcting for the Galactic reddening of $E(B-V)_{\mathrm{Gal}}=0.026 \mathrm{mag}$ with $R_{V}=3.1$ and the host galaxy reddening of $E(B-V)_{\text {host }}=1.42 \pm 0.04$ mag with $R_{V}=$ $1.48 \pm 0.06$, we derive the $B$ - and $V$-band absolute magnitudes to be $-19.10 \pm 0.20$ and $-19.06 \pm 0.17 \mathrm{mag}$, respectively. The magnitudes in other bands are listed in Table 10.

To compare with other SNe Ia, we need to normalize the derived absolute magnitudes of SN 2006X to a nominal lightcurve shape, or $\Delta m_{15}$ value. Phillips (1993) proposed a relation between $\Delta m_{15}$ and the peak luminosity of SNe Ia, and there are now several different versions available in the literature (e.g., Hamuy et al. 1996; Phillips et al. 1999; Altavilla et al. 2004). Based on a large sample of SNe Ia, Prieto et al. (2006) updated the $M_{\max }-\Delta m_{15}$ relation (see their Table 3 ), which we adopt to normalize the luminosity of SN 2006X. An alternative luminosity correction method was proposed by Wang et al. (2005), who introduced a postmaximum color parameter $\Delta C_{12}$ that correlates well with the maximum luminosity of SNe Ia. The relevant correction coefficients are taken from Table 3 in Wang et al. (2006d).

The normalized luminosities (to $\Delta m_{15}=1.1 \mathrm{mag}$ ) of SN 2006X from the two methods are consistent with each other and are listed in Table 10. The normalized luminosity of SN 2006X is consistent with those of the fiducial SN Ia in the $U, B, V$, and $R$ bands, while it is fainter by $\sim 0.2-0.3 \mathrm{mag}$ in the $I, J$, and $K$ bands. This may be partly due to the more pronounced spectral features in the red, such as the broader and deeper absorption trough of the Ca II NIR triplet in SN 2006X than in other SNe Ia, as discussed in $\S 4.1$.

The measured luminosities for SN 2006X from the Cepheid distance to its host galaxy allow us to determine the Hubble constant via the formula $H_{0}=10^{0.2(M+25-\alpha)}$, where $M$ is the absolute magnitude of SN 2006X and $\alpha$ is the zero point defined by Hubble flow SNe Ia. Using the normalized luminosities calibrated from the $\Delta C_{12}$ method and the zero point determined

TABLE 10

Absolute Magnitudes of SN 2006X at Maximum Light

\begin{tabular}{|c|c|c|c|c|c|c|c|}
\hline Method & $M_{U}$ & $M_{B}$ & $M_{V}$ & $M_{R}$ & $M_{I}$ & $M_{J}$ & $M_{K}$ \\
\hline 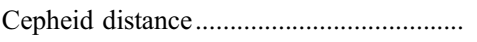 & $-19.63 \pm 0.25$ & $-19.10 \pm 0.20$ & $-19.06 \pm 0.17$ & $-19.02 \pm 0.15$ & $-18.52 \pm 0.18$ & $-18.37 \pm 0.14$ & $-18.23 \pm 0.14$ \\
\hline Normalized to $\Delta m_{15}=1.10 \ldots \ldots \ldots \ldots \ldots \ldots$ & $\ldots$ & $-19.23 \pm 0.26$ & $-19.18 \pm 0.22$ & $-19.13 \pm 0.21$ & $-18.62 \pm 0.23$ & $\ldots$ & $\ldots$ \\
\hline Normalized to $\Delta C_{12}=0.31 \ldots \ldots \ldots \ldots \ldots \ldots$ & $-19.92 \pm 0.30$ & $-19.31 \pm 0.23$ & $-19.22 \pm 0.21$ & $\ldots$ & $-18.63 \pm 0.21$ & $\ldots$ & $\ldots$ \\
\hline
\end{tabular}




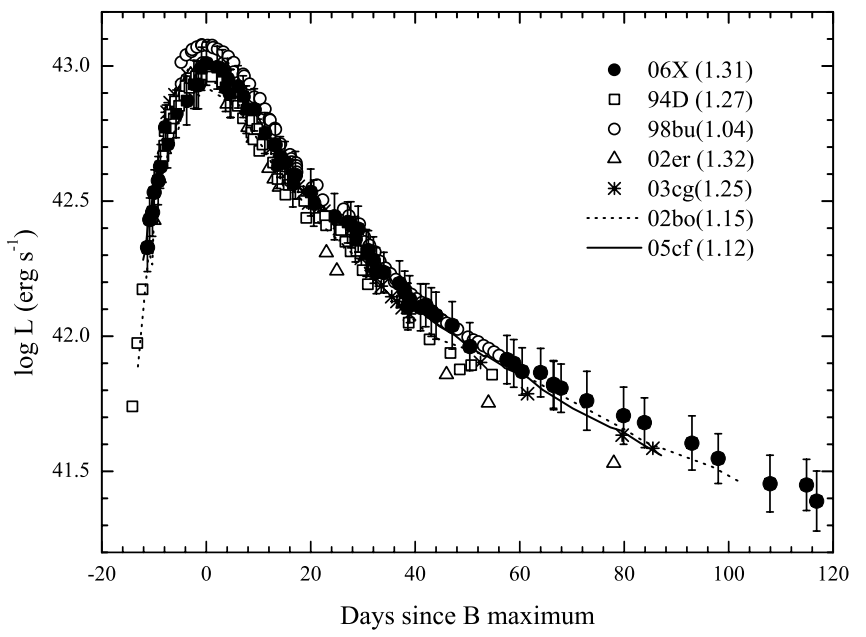

FIG. 20.-Quasi-bolometric light curve of SN 2006X computed from the UBVRIJK light curves. Error bars consist of the uncertainties in the photometry, the distance, and absorption corrections. The quasi-bolometric light curves constructed from SN 1994D, SN 1998bu, SN 2002bo, SN 2002er, SN 2003cg, and SN $2005 \mathrm{cf}$ are overplotted for comparison (see $\S 3.1$ for the data sources). The numbers in parentheses represent the $\Delta m_{15}$ values for the SNe Ia.

by Wang et al. (2006d) from 73 Hubble flow SNe Ia, we derive a Hubble constant (in units of $\mathrm{km} \mathrm{s}^{-1} \mathrm{Mpc}^{-1}$ ) of 71.1, 73.5, 73.8, and 83.9 from the $U, B, V$, and $I$ bands, respectively. Excluding the large value obtained with the peculiar $I$-band data, the average value of $H_{0}$ derived from SN 2006X is $72.8 \pm 8.2 \mathrm{~km} \mathrm{~s}^{-1} \mathrm{Mpc}^{-1}$ (statistical), which is consistent with the estimates from other Cepheid-calibrated SNe Ia (Jha et al. 1999; Riess et al. 2005; Wang et al. 2006d). The statistical error quoted here consists of the uncertainty in the extinction correction and the intrinsic luminosity dispersion of SNe Ia. An analysis of the uncertainty associated with the Cepheid distances is important but is beyond the scope of this paper.

\subsection{Bolometric Light Curve and Nickel Mass}

To better understand the overall properties of SN 2006X, we constructed its quasi-bolometric light curve using our UBVRIJK photometry. For this calculation, we used the normalized passband transmission curves given by Bessell (1990). The integrated flux in each band was approximated by the mean flux multiplied by the effective width of the passband. As we did not get any ultraviolet (below $3200 \AA$ ) data in SN 2006X, we corrected for the contribution of $U V$ flux in our calculation using the recipe given by Suntzeff (1996). Following the procedure in Contardo et al. (2000), we inferred the corrections for the passbands missing between $U$ and $I$ from those SNe Ia that have good observations in all the filters. For the flux beyond $I$ band, we used our own $J K$ data $^{10}$ up to $\sim 80$ days and then applied the Suntzeff IR correction. The resulting quasi-bolometric light curve of SN 2006X is shown in Figure 20, together with those of the comparison SNe Ia.

The reddening-corrected quasi-bolometric luminosity of SN $2006 \mathrm{X}$ is $(1.02 \pm 0.10) \times 10^{43} \mathrm{ergs} \mathrm{s}^{-1}$ at maximum brightness, comparable to that of most comparison SNe Ia but slightly fainter than SN 1998bu. Differences in the peak luminosity are often considered to be caused by variations in the amount of ${ }^{56} \mathrm{Ni}$

\footnotetext{
10 The missing $H$-band data may not impact significantly the construction of the quasi-bolometric light curve, as the flux contribution in $H$ inferred from the SNe Ia with good observations in NIR bands (e.g., SN 2001el; Krisciunas et al. 2003 ) is generally found to be smaller than $3 \%$ of the total flux before 80 days after $B$ maximum.
}

TABLE 11

Relevant Parameters for SN 2006X and Its Host Galaxy

\begin{tabular}{|c|c|c|}
\hline Parameter & Value & Source \\
\hline \multicolumn{3}{|c|}{ SN 2006X Photometric Parameters } \\
\hline 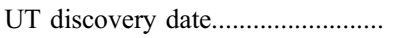 & 2006 Feb 7.15 & 1 \\
\hline Epoch of $B$ maximum...................... & $2,453,786.17 \pm 0.35$ & 2 \\
\hline 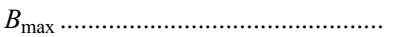 & $15.40 \pm 0.03$ & 2 \\
\hline 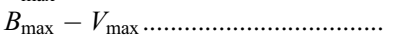 & $1.36 \pm 0.04$ & 2 \\
\hline 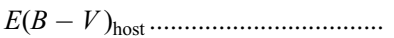 & $1.42 \pm 0.04$ & 2 \\
\hline 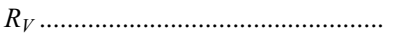 & $1.48 \pm 0.06$ & 2 \\
\hline$M_{\max }^{B} \ldots \ldots \ldots \ldots \ldots \ldots \ldots \ldots \ldots \ldots \ldots \ldots$ & $-19.10 \pm 0.20$ & 2 \\
\hline 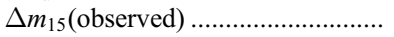 & $1.17 \pm 0.05$ & 2 \\
\hline$\Delta m_{15}$ (true) & $1.31 \pm 0.05$ & 2 \\
\hline 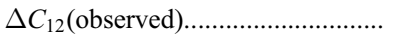 & $1.83 \pm 0.05$ & 2 \\
\hline 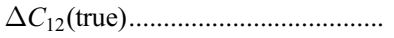 & $0.42 \pm 0.06$ & 2 \\
\hline Late-time $B$ decline rate ................. & $0.92 \pm 0.05 \mathrm{mag}(100 \text { days })^{-1}$ & 2 \\
\hline$H_{0}$ & $72.8 \pm 8.2 \mathrm{~km} \mathrm{~s}^{-1} \mathrm{Mpc}^{-1}$ & 2 \\
\hline$L_{\text {bol }}^{\max } \ldots \ldots \ldots \ldots$ & $(1.02 \pm 0.10) \times 10^{43} \mathrm{ergs} \mathrm{s}^{-1}$ & 2 \\
\hline 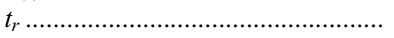 & $18.2 \pm 0.9$ days & 2 \\
\hline 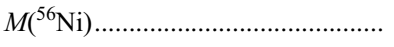 & $0.50 \pm 0.05 M_{\odot}$ & 2 \\
\hline
\end{tabular}

SN 2006X Spectroscopic Parameters

\begin{tabular}{|c|c|}
\hline 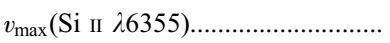 & $\sim 15500 \mathrm{~km} \mathrm{~s}^{-1}$ \\
\hline 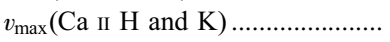 & $\sim 20500 \mathrm{~km} \mathrm{~s}^{-1}$ \\
\hline 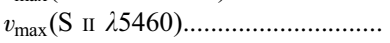 & $\sim 11800 \mathrm{~km} \mathrm{~s}^{-1}$ \\
\hline 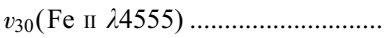 & $\sim 10300 \mathrm{~km} \mathrm{~s}^{-1}$ \\
\hline 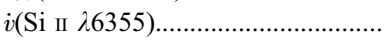 & $123 \pm 10 \mathrm{~km} \mathrm{~s}^{-1}$ \\
\hline$R(\mathrm{Si}$ п) & $0.12 \pm 0.06$ \\
\hline
\end{tabular}

\begin{tabular}{l}
2 \\
2 \\
2 \\
2 \\
2 \\
2 \\
\hline 3 \\
3 \\
4 \\
3
\end{tabular}

\begin{tabular}{|c|c|c|}
\hline 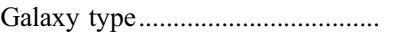 & Sbc, LINER/H II & 3 \\
\hline$E(B-V)_{\mathrm{Gal}} \cdots \cdots \cdots \cdots \cdots \cdots \cdots \cdots \cdots \cdots \cdots \cdots \cdots \cdots \cdots \cdots \cdots \cdots$ & 0.026 & 3 \\
\hline 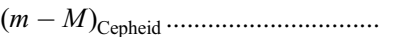 & $30.91 \pm 0.14$ & 4 \\
\hline 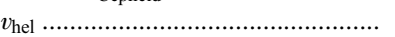 & $1557 \mathrm{~km} \mathrm{~s}^{-1}$ & 3 \\
\hline
\end{tabular}

REFERENCES.-(1) Suzuki \& Migliardi 2006; (2) this paper; (3) NASA Extragalactic Database; (4) Freedman et al. 2001.

synthesized during the explosion. The "bump" feature at $t=$ 20-32 days is more prominent in SN 2006X than in other comparison $\mathrm{SNe}$. This is caused by the more pronounced shoulder and "second maximum" seen in the $R, I$, and NIR bands in SN 2006X. We also note that the contrast between the peak and tail luminosities of SN 2006X is the smallest among the SNe in the comparison: it has a peak luminosity in the middle of the group, but the highest tail luminosity. Efficiency of the trapping of the gamma rays and the positrons from ${ }^{56} \mathrm{Co}$ decay (Milne et al. 2001), as well as small-scale light echoes or ejecta-CSM interaction, may account for this difference.

The radioactive ${ }^{56} \mathrm{Ni}$ synthesized during an $\mathrm{SN}$ Ia explosion is the primary physical parameter determining the peak luminosity, the light-curve width, and the spectroscopic/color evolution of the event (e.g., Kasen \& Woosley 2007). One method to estimate the synthesized ${ }^{56} \mathrm{Ni}$ mass is by assuming that the luminosity at maximum equals the instantaneous energy deposition rate from the radioactive decay, the so-called Arnett law (Arnett 1982; Arnett et al. 1985; Branch 1992). Following Stritzinger \& Leibundgut (2005), the maximum luminosity produced by the radioactive ${ }^{56} \mathrm{Ni}$ can be written as

$$
L_{\max }=\left(6.45 e^{-t_{r} / 8.8}+1.45 e^{-t_{r} / 111.3}\right)\left(\frac{M_{\mathrm{Ni}}}{M_{\odot}}\right) \times 10^{43} \operatorname{ergs~s}^{-1}
$$


where $t_{r}$ is the rise time of the light curve (e.g., the time spent by the $\mathrm{SN}$ from the explosion to the $B$-band maximum) and $M_{\mathrm{Ni}}$ is the ${ }^{56} \mathrm{Ni}$ mass (in units of the solar mass $M_{\odot}$ ). Assuming that the luminosity of an SN Ia evolves as an expanding fireball at very early phase (Goldhaber 1998), the rise time $t_{r}$ can be derived from the relation (Riess et al. 1999)

$$
L(t)=\varepsilon\left(t+t_{r}\right)^{2},
$$

where $t$ is the elapsed time since maximum and $\varepsilon$ is the parameter describing the rising rate of the luminosity. Using very early photometric data in the $R$ band (including the earliest unfiltered data from IAU Circ. 8667), we derive the rise time to the $B$ maximum as $18.2 \pm 0.9$ days. This is slightly shorter than the average rise time of $19.5 \pm 0.2$ days for a normal SN Ia with $\Delta m_{15}=1.1 \mathrm{mag}$ ( Riess et al. 1999; but for the study of a possible dichotomy of the rise time in SNe Ia see Strovink 2007) but is consistent with that derived for SN 2002bo $\left(t_{r}=17.9 \pm\right.$ 0.5 days; Benetti et al. 2004) and SN 2005cf $\left(t_{r}=18.6 \pm 0.4\right.$ days; Pastorello et al. 2007).

With the peak quasi-bolometric luminosity and the rise time derived for SN $2006 \mathrm{X}$, we derive a ${ }^{56} \mathrm{Ni}$ mass of $0.50 \pm 0.05 M_{\odot}$. This is within the reasonable range of ${ }^{56} \mathrm{Ni}$ masses of normal $\mathrm{SNe}$ Ia. The quoted error bar includes uncertainties in the rise time and bolometric luminosity.

Table 11 lists all the important parameters derived for SN 2006X in the previous sections, as well as the parameters relevant to its host galaxy M100.

\section{DISCUSSION AND CONCLUSIONS}

In this paper we present extensive optical and NIR photometry, as well as optical spectroscopy, of the nearby SN Ia SN 2006X in NGC 4321 (M100). Our observations indicate that SN 2006X is a highly reddened object with an $R_{V}$ value much smaller than the canonical 3.1 for average Galactic dust and has the highest expansion velocity ever published for an SN Ia.

Compared with other SNe Ia included in this paper, SN 2006X has a broader light curve in the $U$ band, a more prominent "bump" feature in the $V$ band, a more pronounced shoulder in the $R$ band, and a brighter second maximum in the $I J K$ bands. Relative to the peak brightness, SN 2006X has a higher luminosity in the UBVRIJK light curves during the nebular phase than other comparison $\mathrm{SNe}$ Ia. The $B$-band decline rate at late times, $\beta=$ $0.92 \pm 0.05$ mag $(100 \text { days })^{-1}$, is much smaller than that of most normal SNe Ia. We note that other SNe Ia with high expansion velocities, such as SN 1984A and SN 2002bo, also exhibit relatively small late-time decline rates. Thus, slow late-time decline in the blue bands may be common for rapidly expanding SNe Ia.

The most notable features in the color curves of SN 2006X are a plateau in the $U-B$ color after $B$ maximum and an obvious deviation from the Lira-Phillips relation at $t=30-90$ days. After SN 2006X reached its reddest color in $B-V$ at $t \approx 30$ days, it became progressively bluer at a much faster pace than expected from the Lira-Phillips relation. We thus suggest that the LiraPhillips relation should be applied with caution to any individual SN Ia.

We use a global color curve fitting method and the empirical relations between $\Delta m_{15}$, the peak colors $C_{\max }$, and the postmaximum color $\Delta C_{12}$ to determine the reddening and extinction of SN 2006X. This yields an average estimate of the host galaxy reddening for SN 2006X, $E(B-V)_{\text {host }}=1.42 \pm 0.04$ mag. Assuming that the absorption by dust follows the analytical model proposed by Cardelli et al. (1989), we obtain $R_{V}=1.48 \pm 0.06$.
The low $R_{V}$ value suggests that the dust around SN 2006X is quite different from that observed in the Milky Way, perhaps of a different origin than normal interstellar medium dust. This is also demonstrated by the relatively smaller EW of the interstellar $\mathrm{Na}$ I $\mathrm{D}$ absorption in the spectra. Further evidence against conventional interstellar dust for SN 2006X is that the polarized spectrum of SN 2006X is significantly different from that of extinguished Galactic stars (Wang et al. 2006a). It is worth pointing out that most highly reddened known $\mathrm{SNe}$ Ia, with $E(B-$ $V)_{\text {host }}>0.5 \mathrm{mag}$, tend to have $R_{V}$ values smaller than 3.1. This suggests that the dust affecting some $\mathrm{SNe}$ Ia may be quite different from that observed in the Galaxy.

We caution, however, that SN 2006X may have a peculiar (unknown) intrinsic color evolution, as suggested by the deviation from the Lira-Phillips relation in the $B-V$ color. If so, it would be difficult to estimate the host galaxy reddening toward SN 2006X and to study its photometric properties.

Spectra of SN 2006X reveal high expansion velocities, based on the IMEs and the iron group elements. SN 2006X evolves in a manner similar to that of other rapidly expanding events such as SN 1984A and SN 2002bo. At early times, these high- $v_{\text {exp }}$ objects have much stronger $\mathrm{Si}$ II $\lambda 6355, \mathrm{Ca}$ II H and K, and Ca II NIR triplet lines than other SNe Ia. At late times, spectra of SN 2006X show a relatively bluer overall continuum, especially at $\lambda<4500 \AA$, than the comparison SNe. This is consistent with the bluer $U-B$ and $B-V$ colors of SN 2006X than the other SNe Ia at similar epochs and suggests an additional input of energy, probably a light echo.

To explain the high-velocity features of some SNe Ia, Benetti et al. (2004) proposed a scenario in which the burning to IMEs extends farther out into the outermost layers than in normal SNe Ia. This model produces the IMEs at higher velocities, but with no additional ${ }^{56} \mathrm{Ni}$ production, which provides a plausible explanation for most of the spectral features seen in SN 2006X. The above scenario should account not only for the high-velocity feature of the IMEs but also for that of the iron group elements at earlier phases of SN evolution.

On the other hand, we propose that SN 2006X may be produced in a progenitor environment having abundant CSM. In this scenario, the high-velocity features shown in the spectra are formed due to the density increase caused by interaction between the supernova ejecta and the surrounding material of the progenitor system, which could be an accretion disk, a filled Roche lobe, or a common envelope (Gerardy et al. 2004).

The CSM dust, quite possibly associated with the CSM produced by the progenitor system (Patat et al. 2007), may be the source of a local light echo that could account for the late-time extra energy (beyond radioactive decay) seen at short wavelengths. SN 2006X-like events may actually represent a subclass of SNe Ia with distinct features of spectroscopy, photometry, polarization, and even extinction, and the presence of significant CSM dust may be one of the possible explanations (X. Wang et al. 2008 , in preparation). There is also a possibility that the detection of CSM in SN 2006X is just tied to the particular viewing angle. Obviously, much further research, both observational and theoretical, is needed on this problem.

Some of the data presented here were obtained at the W. M. Keck Observatory, which is operated as a scientific partnership among the California Institute of Technology, the University of California, and the National Aeronautics and Space Administration (NASA). The Observatory was made possible by the generous financial support of the W. M. Keck Foundation. We 
thank the BAO, Keck, and Lick Observatory staffs for their assistance with the observations. This research was supported by NSF grant AST 06-07485, the TABASGO Foundation, and the National Natural Science Foundation of China (NSFC grant 10673007) and the Basic Research Funding at Tsinghua University (JCqn2005036). KAIT was made possible by generous donations from Sun Microsystems, Inc., the Hewlett-Packard Company, AutoScope Corporation, Lick Observatory, the University of California, and the Sylvia \& Jim Katzman Foundation.
The CTIO $1.3 \mathrm{~m}$ telescope is operated by the Smart and Moderate Aperture Research Telescope System (SMARTS) Consortium. We are particularly grateful for the scheduling flexibility of SMARTS. A. V. F. thanks the Aspen Center for Physics, where he participated in the workshop on "Supernovae as Cosmological Distance Indicators" while this paper was nearing completion. We made use of the NASA/IPAC Extragalactic Database (NED), which is operated by the Jet Propulsion Laboratory, California Institute of Technology, under contract with NASA.
Altavilla, G., et al. 2004, MNRAS, 349, 1344

Arnett, W. D. 1982, ApJ, 253, 785

Arnett, W. D., Branch, D., \& Wheeler, J. C. 1985, Nature, 314, 337

Astier, P., et al. 2006, A\&A, 447, 31

Barbon, B., Benetti, S., Cappellaro, E., Rosino, L., \& Turrato, M. 1990, A\&A, 237,79

Barbon, B., Rosino, L., \& Iijima, T. 1989, A\&A, 220, 83

Barris, B. J., et al. 2004, ApJ, 602, 571

Benetti, S., et al. 2004, MNRAS, 348, 261 2005, ApJ, 623, 1011

Bessell, M. S. 1990, PASP, 102, 1181

Branch, D. 1992, ApJ, 392, 35

Branch, D., et al. 2005, PASP, 117, 545 2006, PASP, 118, 560

Cappellaro, E., et al. 2001, ApJ, 549, L215

Cardelli, J. A., Clayton, G. C., \& Mathis, J. S. 1989, ApJ, 345, 245

Contardo, G., Leibundgut, B., \& Vacca, W. D. 2000, A\&A, 359, 876

Cousins, A. W. J. 1981, S. African Astron. Obs. Circ., 6, 4

Eisenstein, D. J., et al. 2005, ApJ, 633, 560

Elias-Rosa, N., et al. 2006, MNRAS, 369, 1880 (ER06)

Elmhamdi, A., Chugai, N. N., \& Danziger, I. J. 2003, A\&A, 404, 1077

Ferarrese, L., et al. 1997, ApJ, 475, 853

Filippenko, A. V. 1982, PASP, 94, 715 1997, ARA\&A, 35, 309

2005, in White Dwarfs: Cosmological and Galactic Probes, ed. E. M.

Sion, S. Vennes, \& H. L. Shipman (Dordrecht: Springer), 97

Filippenko, A. V., Li, W., Treffers, R. R., \& Modjaz, M. 2001, in ASP Conf.

Ser. 246, Small Telescope Astronomy on Global Scales, ed. B. Paczyński,

W.-P. Chen, \& C. Lemme (San Francisco: ASP), 121

Foley, R. J., et al. 2003, PASP, 115, 1220

Freedman, W. L., et al. 2001, ApJ, 553, 47

Garavini, G., et al. 2007, A\&A, 471, 527

Gerardy, C., et al. 2004, ApJ, 607, 391

Goldhaber, G. 1998, BAAS, 30, 1325

Hamuy, M., Phillips, M. M., Suntzeff, N. B., Schommer, R. A., Maza, J., \& Aviles, R. 1996, AJ, 112, 2398

Ho, L. C., Filippenko, A. V., \& Sargent, W. L. W. 1997, ApJS, 112, 315

Iben, I., Jr., \& Tutukov, A. 1984, ApJS, 54, 335

Jeffery, D. J., \& Branch, D. 1990, in Supernovae, Jerusalem Winter School for

Theoretical Physics, ed. J. C. Wheeler, T. Piran, \& S. Weinberg (Singapore: World Scientific), 149

Jha, S., Riess, A. G., \& Kirshner, R. P. 2007, ApJ, 659, 122

Jha, S., et al. 1999, ApJS, 125, 73

Johnson, H. L., Iriarte, B., Mitchell, R. I., \& Wisniewskj, W. Z. 1966, Comm.

Lunar Planet. Lab., 4, 99

Kasen, D. 2006, ApJ, 649, 939

Kasen, D. \& Woosley, S. E. 2007, ApJ, 656, 661

Knop, R. A., et al. 2003, ApJ, 598, 102

Krisciunas, K., Prieto, J. L., Garnavich, P. M., Riley, Jessica-Lynn, G., Rest, A., Stubbs, C., \& McMillan, R. 2006, AJ, 131, 1639

Krisciunas, K., et al. 2000, ApJ, 539, 658

2003, AJ, 125, 166

2004, AJ, 128,3034

Landolt, A. U. 1992, AJ, 104, 340

Lentz, E. J., Baron, E., Branch, D., \& Hauschildt, P. H. 2001, ApJ, 557, 266

Lentz, E. J., Baron, E., Branch, D., Hauschildt, P. H., \& Nugent, P. E. 2000, ApJ, 530, 966

\section{REFERENCES}

Li, W., et al. 2001, PASP, 113, 1178

Lira, P. 1995, Masters thesis, Univ. Chile

Mazzali, P. A., Lucy, L. B., Danziger, I. J., Couiffes, C., Cappellaro, E., \& Turatto, M. 1993, A\&A, 269, 423

Miller, J. S., \& Stone, R. P. S. 1993, Lick Obs. Tech. Rep. 66

Milne, P. A., The, L. S., \& Leising, M. D. 2001, ApJ, 559, 1019

Munari, U., \& Zwitter, T. 1997, A\&A, 318, 269

Nomoto, K., Iwamoto, K., \& Kishimoto, N. 1997, Science, 276, 1378

Nugent, P., et al. 1995, ApJ, 455, L147

Oke, J. B., et al. 1995, PASP, 107, 375

Pastorello, A., et al. 2007, MNRAS, 376, 1301

Patat, F. 2006, MNRAS, 369, 1949

Patat, F., Benetti, S., Cappellaro, E., Danziger, I. J., Della Valle, M., Mazzali, P. A., \& Turatto, M. 1996, MNRAS, 278, 111

Patat, F., et al. 2007, Science, 317, 924

Perlmutter, S., et al. 1999, ApJ, 517, 565

Persson, S. E., Murphy, D. C., Krzeminski, W., Roth, M., \& Rieke, M. J. 1998, AJ, 116, 2475

Phillips, M. M. 1993, ApJ, 413, L105

Phillips, M. M., et al. 1999, AJ, 118, 1766

Pignata, G., et al. 2004, MNRAS, 355, 178

Prieto, J. L., Rest, A., \& Suntzeff, N. B. 2006, ApJ, 647, 501

Quimby, R., Brown, P., \& Gerardy, C. 2006, CBET, 393, 1

Reindl, B., Tammann, G. A., Sandage, A., \& Saha, A. 2005, ApJ, 624, 532

Richmond, M. W., et al. 1995, AJ, 109, 2121

Riess, A. G., Press, W. H., \& Kirshner, R. P. 1996, ApJ, 473, 588

Riess, A. G., et al. 1998, AJ, 116, 1009 1999, AJ, 118, 2675

2004, ApJ, 607, 665

2005, ApJ, 627, 579

2007, ApJ, 659, 98

Salvo, M. E., et al. 2001, MNRAS, 321, 254

Schlegel, D. J., Finkbeiner, D. P., \& Davis, M. 1998, ApJ, 500, 525

Spergel, D. N., et al. 2003, ApJS, 148, 175 2007, ApJS, 170, 377

Spyromilio, J., Gilmozzi, R., Sollerman, J., Leibundgut, B., Fransson, C., \&

Cuby, J. G. 2004, A\&A, 426, 547

Stanishev, V., et al. 2007, A\&A, 469, 645

Stetson, P. B. 1987, PASP, 99, 191

Stritzinger, M., \& Leibundgut, B. 2005, A\&A, 431, 423

Stritzinger, M., et al. 2002, AJ, 124, 2100

Strovink, M. 2007, ApJ, 671, 1084

Suntzeff, N. B. 1996, in Supernova and Supernova Remnants, ed. R. McCray \&

Z. R. Wang (Cambridge: Cambridge Univ. Press), 41

Suzuki, S., \& Migliardi, M. 2006, IAU Circ., 8667

Tonry, J. L., et al. 2003, ApJ, 594, 1

Tripp, R. 1997, A\&A, 325, 871

Wang, L. 2005, ApJ, 635, L33

Wang, L., Baade, D., Patat, F., \& Wheeler, J. C. 2006a, CBET, 396, 2

Wang, L., et al. 2006b, ApJ, 641, 50 . 2006c, ApJ, 653, 490

Wang, X., Wang, L., Zhou, X., Lou, Y., \& Li, Z. 2005, ApJ, 620, L87

Wang, X., et al. 2006d, ApJ, 645, 488 2008, ApJ, in press (arXiv:0711.2570)

Webbink, R. F. 1984, ApJ, 277, 355

Wood-Vasey, W. M., et al. 2007, ApJ, 666, 694 